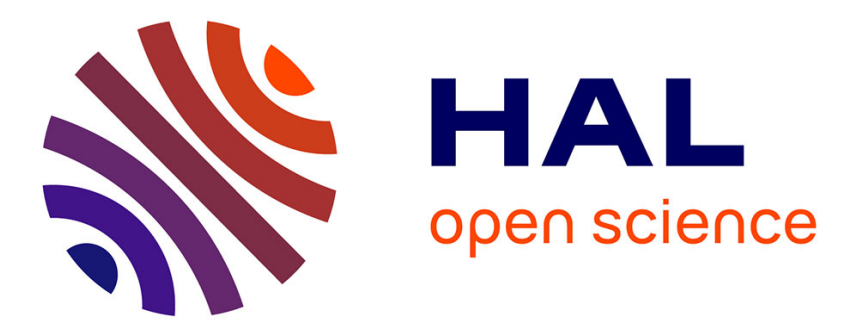

\title{
Thermomechanics of shells undergoing phase transition
}

V. A. Eremeyev, W. Pietraszkiewicz

\section{To cite this version:}

V. A. Eremeyev, W. Pietraszkiewicz. Thermomechanics of shells undergoing phase transition. Journal of the Mechanics and Physics of Solids, 2011, 59 (7), pp.1395-1412. hal-00835667

\section{HAL Id: hal-00835667 https://hal.science/hal-00835667}

Submitted on 19 Jun 2013

HAL is a multi-disciplinary open access archive for the deposit and dissemination of scientific research documents, whether they are published or not. The documents may come from teaching and research institutions in France or abroad, or from public or private research centers.
L'archive ouverte pluridisciplinaire HAL, est destinée au dépôt et à la diffusion de documents scientifiques de niveau recherche, publiés ou non, émanant des établissements d'enseignement et de recherche français ou étrangers, des laboratoires publics ou privés. 


\title{
Thermomechanics of shells undergoing phase transition
}

\author{
V.A. Eremeyev ${ }^{\mathrm{a}, \mathrm{b}}$, W. Pietraszkiewicz ${ }^{\mathrm{c}, *}$ \\ ${ }^{a}$ Martin Luther Universiy Halle Wittenberg, Halle (Saale), Germany \\ ${ }^{\mathrm{b}}$ South Scientific Center, RASci E South Federal University, Rostov on Don, Russia \\ 'Institute of Fluid Flow Machinery, PASci, Gdansk, Poland
}

\begin{abstract}
A B S T R AC T
The resultant, two dimensional thermomechanics of shells undergoing diffusionless, displacive phase transitions of martensitic type of the shell material is developed. In particular, we extend the resultant surface entropy inequality by introducing two temperature fields on the shell base surface: the referential mean temperature and its deviation, with corresponding dual fields: the referential entropy and its deviation. Additionally, several extra surface fields related to the deviation fields are introduced to assure that the resultant surface entropy inequality be direct implication of the entropy inequality of continuum thermomechanics. The corresponding constitutive equations for thermoelastic and thermoviscoelastic shells of differential type are worked out Within this formulation of shell thermomechanics, we also derive the thermodynamic continuity condition along the curvilinear phase interface and propose the kinetic equation allowing one to determine position and quasistatic motion of the interface relative to the base surface. The theoretical model is illustrated by two axisymmetric numerical examples of stretching and bending of the circular plate undergoing phase transition within the range of small deformations.
\end{abstract}

\section{Introduction}

Phase transition (PT) phenomenon in continuous media originally described by Gibbs (1928) was developed in a number of papers summarised in several recent books for example by Grinfeld (1991), Gurtin (1993, 2000), Romano (1993), Sun (2002), Bhattacharya (2003), Fischer (2004), Abeyaratne and Knowles (2006), Lagoudas (2008), and Berezovski et al. (2008). In this approach one assumes existence of the sharp phase interface being a sufficiently regular surface dividing different material phases. The position and motion of the phase interface itself is among the most discussed issues in the field. In the literature many model one-dimensional (1D) problems were analysed theoretically, numerically and experimentally which adequately described behaviour of bars, rods, and beams made of martensitic materials.

Experiments on shape memory alloys and other materials undergoing PT are often performed with thin-walled samples such as thin strips, rectangular plates or thin tubes, see Li and Sun (2002), He and Sun (2009a,b, 2010a,b), and Sun (2002) among others. One would expect that two-dimensional (2D) thermomechanics describing the behaviour of thin-walled structural elements made of materials undergoing PT which is based on the theory of shells was developed long ago. But this is not the case. To our best knowledge a simple 2D mechanical model of PT in thin films was proposed by Bhattacharya and James (1999), James and Rizzoni (2000), and Shu (2000), see also Bhattacharya (2003) and Miyazaki et al. (2009).

\footnotetext{
${ }^{*}$ Corresponding author. Tel.: +48585525677; fax: +48583416144 .

E mail addresses: eremeyev.victor@gmail.com (V.A. Eremeyev), pietrasz@imp.gda.pl (W. Pietraszkiewicz).
} 
The model consists of the Cosserat membrane with one director, but without taking into account bending rigidity of the membrane. Alternative simple models of PT in biomembranes were proposed by Boulbitch (1999), Agrawal and Steigmann (2008), and Elliott and Stinner (2010).

The non-linear equilibrium conditions of elastic shells undergoing PT of martensitic type were formulated by Eremeyev and Pietraszkiewicz (2004) and Pietraszkiewicz et al. (2007) within the dynamically exact and kinematically unique theory of shells developed by Libai and Simmonds $(1983,1998)$ and Chróścielewski et al. (2004). This version of the non-linear theory of shells has the structure of the classical Cosserat surface with the translation vector $\boldsymbol{u}$ and rotation tensor $\boldsymbol{Q}$ fields as the only independent variables. By analogy to the 3D case, the two-phase shell was regarded in Eremeyev and Pietraszkiewicz (2004) and Pietraszkiewicz et al. (2007) as the Cosserat surface consisting of two material phases divided by a sufficiently smooth surface curve. Existence of such a curve was confirmed by several experiments on thin-walled samples. For such a general shell model the first 2D thermomechanical model of PT has recently been worked out by Eremeyev and Pietraszkiewicz (2009).

In this paper we develop the general non-linear thermomechanics of the resultant Cosserat-type shells undergoing diffusionless (displacive) phase transitions of martensitic type. In particular, we discuss the thermodynamic condition allowing one to determine position and quasistatic motion of the phase interface on the deformed shell base surface. Here we use extended thermodynamics of shells based on the introduction of two temperature fields. The theoretical model is illustrated by example of stretching and bending of the circular plate undergoing phase transition in the case of small deformations.

\section{Kinematics}

In the undeformed placement the shell-like body is represented by the base surface $M$ described by the position vector $\boldsymbol{x}\left(\theta^{\alpha}\right)$, and orientation of $M$ is defined by the unit normal vector $\boldsymbol{\eta}\left(\theta^{\alpha}\right)$, with $\left\{\theta^{\alpha}\right\}, \alpha \quad 1,2$, the surface curvilinear coordinates.

Within the dynamically exact and kinematically unique theory of shells summarised in Libai and Simmonds (1998), Chróścielewski et al. (2004), Eremeyev and Zubov (2008), in the deformed placement the shell is represented by the position vector $\boldsymbol{y} \quad \chi(\boldsymbol{x})$ of the deformed material base surface $N \quad \chi(M)$ with attached three directors (d $\left.\boldsymbol{d}_{\alpha}, \boldsymbol{d}\right)$ such that

$$
\boldsymbol{y} \quad \boldsymbol{x}+\boldsymbol{u}, \quad \boldsymbol{d}_{\alpha} \quad \boldsymbol{Q} \boldsymbol{x}_{, \alpha}, \quad \boldsymbol{d} \quad \boldsymbol{Q} \boldsymbol{\eta},
$$

where $\chi$ is the surface deformation function, $\boldsymbol{u} \in E$ the translation vector of $M$, and $\boldsymbol{Q} \in S O$ (3) the proper orthogonal tensor, $\mathbf{Q}^{T} \quad \mathbf{Q}^{-1}$, det $\boldsymbol{Q}+1$, representing the work-averaged gross rotation of the shell cross sections from their undeformed shapes described by $\left(\boldsymbol{x}_{,}, \boldsymbol{\eta}\right)$.

In the shell undergoing phase transition it is assumed that above some level of deformation different material phases $A$ and $B$ may appear in different complementary subregions $N_{A}$ and $N_{B}$ separated by the curvilinear phase interface $D \in N$. For a piecewise differentiable mapping $\chi$ we can introduce on $M$ a singular image curve $C \chi^{-1}(D)$ separating the corresponding image regions $M_{A} \quad \chi^{-1}\left(N_{A}\right)$ and $M_{B} \quad \chi^{-1}\left(N_{B}\right)$. The position vectors of $C$ and $D$ are related by $\boldsymbol{x}_{C}(s) \quad \chi^{-1}\left(\boldsymbol{y}_{C}(s)\right)$, where $s$ is the arc length parameter along $C$.

Let us consider a one-parameter family of shell deformations

$$
\begin{array}{llllll}
\boldsymbol{y}(\boldsymbol{x}, t) & \boldsymbol{x}+\boldsymbol{u}(\boldsymbol{x}, t), & \boldsymbol{d}_{\alpha}(\boldsymbol{x}, t) & \boldsymbol{Q}(\boldsymbol{x}, t) \boldsymbol{x}, \alpha, & \boldsymbol{d}(\boldsymbol{x}, t) & \boldsymbol{Q}(\boldsymbol{x}, t) \boldsymbol{\eta}(\boldsymbol{x}),
\end{array}
$$

where $t$ is a time-like scalar parameter such that $t \quad 0$ corresponds to the undeformed placement and $t$ to the deformed one. Then $v \quad \dot{\boldsymbol{u}}$ is the virtual translation vector, and $\boldsymbol{\omega}$ ax $\left(\dot{\mathbf{Q}} \mathbf{Q}^{T}\right)$ the virtual rotation vector, where ax(...) is the axial vector associated with the skew tensor (..), (..) $d / d t(\ldots)$, while $V \quad \dot{\boldsymbol{x}}_{C} \cdot \boldsymbol{v}$ is the virtual translation component in the exterior normal direction of the phase curve $C, \boldsymbol{v} \in T_{X} M$ is the unit external normal vector to $C$, and $\boldsymbol{v} \cdot \boldsymbol{\eta} \quad 0$.

In the general resultant theory of shells considered here the following two strain measures are introduced, see Chróścielewski et al. (2004), Eremeyev and Pietraszkiewicz (2004, 2006), and Pietraszkiewicz et al. (2005):

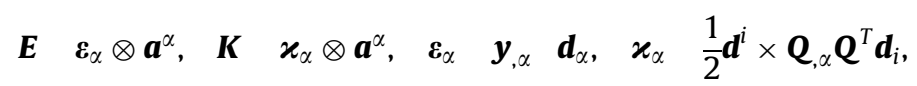

where $\left(\boldsymbol{a}^{\alpha}, \boldsymbol{\eta}\right)$ and $\left(\boldsymbol{d}^{i}\right)$ are bases reciprocal to the base $\left(\boldsymbol{x}_{, \alpha}, \boldsymbol{\eta}\right)$ and the base $\left(\boldsymbol{d}_{\alpha}, \boldsymbol{d}\right)$, respectively.

The curvilinear phase interfaces in shells can be either coherent or incoherent in rotations, see Eremeyev and Pietraszkiewicz (2004). For the coherent interface both fields $\boldsymbol{y}$ (or $\boldsymbol{u}$ ) and $\boldsymbol{Q}$ are supposed to be continuous at $C$ and the kinematic compatibility conditions along $C$ become, see Eremeyev and Pietraszkiewicz (2004), Eqs. (31) and (34),

$$
\llbracket \boldsymbol{v} \rrbracket+V \llbracket \boldsymbol{F} \boldsymbol{v} \rrbracket \quad \mathbf{0}, \quad \llbracket \boldsymbol{\omega} \rrbracket+V \llbracket \boldsymbol{K} \boldsymbol{v} \rrbracket \quad \mathbf{0},
$$

where the expression $\llbracket \ldots \rrbracket \quad(\ldots)_{B} \quad(\ldots)_{A}$ means the jump at $C$.

The phase interface is called incoherent in rotations if only $\boldsymbol{y}$ (or $\boldsymbol{u}$ ) is continuous at $C$ but the continuity of $\boldsymbol{Q}$ may be violated. In this case the condition $(4)_{1}$ is still satisfied, but $(4)_{2}$ may be violated, see Eremeyev and Pietraszkiewicz (2004).

\section{Equilibrium equations}

The balance equations and corresponding dynamic boundary conditions of the general non-linear theory of shells can be derived exactly by direct through-the-thickness integration of 3D balance laws of linear and angular momentum of 
continuum mechanics, see Libai and Simmonds (1998), Chróścielewski et al. (2004), and Eremeyev and Pietraszkiewicz (2004). In quasistatic problems discussed here the global equilibrium conditions lead to the local Lagrangian equilibrium equations and the static boundary conditions

$$
\operatorname{Div} \boldsymbol{N}+\boldsymbol{f} \quad \mathbf{0}, \quad \operatorname{Div} \boldsymbol{M}+\operatorname{ax}\left(\boldsymbol{N F} \boldsymbol{F}^{T} \quad \boldsymbol{N N}^{T}\right)+\boldsymbol{c} \quad \mathbf{0} \text { in } M,
$$

$\begin{array}{llllll}\boldsymbol{N} & \boldsymbol{n}^{*} & \mathbf{0}, & \boldsymbol{M} \boldsymbol{v} & \boldsymbol{m}^{*} & \mathbf{0} \text { along } \partial M_{f},\end{array}$

where the tensors $\boldsymbol{N}, \boldsymbol{M} \in E \otimes T_{x} M$ defined on $M$ are the resultant surface stress measures of the 1st Piola-Kirchhoff type, $\boldsymbol{f}, \boldsymbol{c}$ are the resultant surface force and couple vector fields acting on $N \backslash D$, but measured per unit area of $M \backslash C$, while $\boldsymbol{n}^{*}$ and $\boldsymbol{m}^{*}$ are the external boundary resultant force and couple vectors applied along the part $\partial N_{f}$ of $N \quad \chi(M)$, respectively. Additionally, $\boldsymbol{F} \operatorname{Grad} \boldsymbol{y} \quad \boldsymbol{y}_{, \alpha} \otimes \boldsymbol{a}^{\alpha}$ is the surface deformation gradient, $\boldsymbol{F} \in E \otimes T_{x} M$, Div $\boldsymbol{N} \boldsymbol{N}_{, \alpha} \cdot \boldsymbol{a}^{\alpha}$ means the surface divergence of $\boldsymbol{N}$, while ax $\boldsymbol{T}$ is the axial vector of the skew tensor $\boldsymbol{T}^{T} \quad \boldsymbol{T}$.

At the curvilinear phase interface $C$, which is the singular surface curve with regard to the surface stress measures, we obtain the local Lagrangian dynamic compatibility conditions (Chróścielewski et al., 2004),

$$
\llbracket \boldsymbol{N} \boldsymbol{v} \rrbracket \quad \mathbf{0}, \quad \llbracket \boldsymbol{M v} \rrbracket+\llbracket \boldsymbol{y} \times \boldsymbol{N} \boldsymbol{v} \rrbracket \quad \mathbf{0} .
$$

Eqs. (6) are just the local balances of forces and couples at $C$ in the quasistatic deformation process of the interface.

Further we assume that the position vector $\boldsymbol{y}$ is the continuous vector function, i.e. we assume that the following relation holds:

$$
\llbracket \boldsymbol{y} \rrbracket \quad \mathbf{0} \text { at } C \text {. }
$$

This means that we consider the phase interfaces which are coherent in translations, but still incoherent in rotations. Under the condition (7) with (6) 1 we obtain $\llbracket \boldsymbol{y} \times \boldsymbol{N} \boldsymbol{v} \rrbracket \quad \mathbf{0}$ and $(6)_{2}$ becomes $\llbracket \boldsymbol{M v} \rrbracket$

0.

\section{Energy balance equation}

According to Simmonds (1984) and Eremeyev and Pietraszkiewicz (2009), the local resultant 2D thermomechanic balances of energy in the referential description read

$$
\begin{array}{llll}
\rho \dot{\varepsilon} & \rho r & \operatorname{Div} \boldsymbol{q}+\boldsymbol{N} \bullet E^{\circ}+\boldsymbol{M} \bullet \boldsymbol{K}^{\circ} & \text { in } M \backslash C, \\
\boldsymbol{q} \cdot \boldsymbol{v} & q^{*} & 0 & \text { along } \partial M_{h},
\end{array}
$$

where $\varepsilon$ is the internal surface energy (density), $r$ is the internal surface heat supply minus fluxes through the upper and lower shell faces, all per unit mass of $M, \boldsymbol{q} \in T_{x} M$ is the referential surface heat flux vector, $(\cdot)^{\circ} \quad \mathbf{Q}(d / d t)\left[\mathbf{Q}^{T}(\cdot)\right]$ is the corotational time derivative, and the scalar product of two tensors $\boldsymbol{A}, \boldsymbol{B} \in E \otimes T_{x} M$ is defined by $\boldsymbol{A} \bullet \boldsymbol{B} \operatorname{tr}\left(\boldsymbol{A}^{T} \boldsymbol{B}\right)$.

The corresponding local energy balance along $C$ is

$$
V \llbracket \rho \varepsilon \rrbracket+\llbracket \boldsymbol{n}_{v} \cdot \boldsymbol{v} \rrbracket+\llbracket \boldsymbol{m}_{v} \cdot \boldsymbol{\omega} \rrbracket \quad \llbracket \boldsymbol{q} \cdot \boldsymbol{v} \rrbracket \quad 0,
$$

while $\boldsymbol{n}_{v} \quad \boldsymbol{N} \boldsymbol{v}$ and $\boldsymbol{m}_{v} \quad \boldsymbol{M} \boldsymbol{v}$ are the internal contact stress resultant and couple vectors at the arbitrary edge $\partial R$ of $R \quad \chi(\Pi)$.

\section{The entropy inequality}

The referential resultant entropy inequality for the shell can also be derived by direct through-the-thickness integration of the global 3D entropy inequality (The 2nd Law). In the literature there is no agreement which form should take The 2nd Law, see for example the reviews by Muschik et al. (2001) and Muschik (2008). Besides, in various formulations of The 2nd Law different ways of introducing the temperature field are proposed.

Thermodynamics of shells from various points of view was presented for example in Green and Naghdi $(1970,1979)$, Murdoch (1976a,b), Zhilin (1976), Simmonds (1984, 2005, in press), Rubin (2004, 2006), Makowski and Pietraszkiewicz (2002), Eremeyev and Zubov (2008) and Eremeyev and Pietraszkiewicz (2009). In the papers various sets of surface fields responsible for temperature were used and several formulations of the first and second laws of thermodynamics for shells were discussed.

The simplest version of 2D second law of shell thermodynamics was proposed in Murdoch (1976a,b), where the shell base surface was equipped with one temperature field $\theta$ and one corresponding work-conjugate entropy field $\eta$. At the same time, two different temperatures $\Theta_{ \pm}$of the surrounding media are admitted above and below the base surface. The simplified version of Murdoch (1976a,b) with $\Theta_{+} \quad \theta \quad \Theta_{-}$was used in Eremeyev and Pietraszkiewicz (2009) to formulate the resultant 2D 2nd Law along the curvilinear phase interface in the two-phase shell. In Green and Naghdi (1970, 1979), Simmonds (1984, 2005 , in press), and Rubin $(2004,2006)$ the through-the-thickness averaged temperature and its averaged derivative in the thickness direction were used as the independent field variables. Various hypotheses for definition of the surface temperature on the material base surface were discussed in Steinmann and Häsner (2005). In Zhilin (1976) and Eremeyev and Zubov (2008) two temperature fields $\Theta_{+}$and $\Theta_{-}$were used as two independent field variables. In Zhilin (1976) two 2D entropy inequalities were introduced to represent on the shell base surface one entropy inequality used in 3D continuum mechanics. Even more general version of 2D shell thermodynamics was discussed in Makowski and Pietraszkiewicz (2002). 
In the present paper we use on the 3D level the rational thermomechanics proposed by Truesdell and Toupin (1960), in which The 3D 2nd Law is given in the form

$$
\dot{H} \geq J,
$$

where the entropy $H$ and the entropy production $J$ are given in the Clausius-Duhem form

$$
H \quad \iiint_{\mathcal{P}} \mathrm{Q}_{R} \eta d a, \quad J \quad \iiint_{\mathcal{P}} \mathrm{Q}_{R} \frac{\mathrm{r}}{\Theta} d v \iint_{\partial \mathcal{P}} \frac{\mathbf{q} \cdot \mathbf{n}}{\Theta} d a .
$$

In (11), $\mathcal{P}$ is the $3 \mathrm{D}$ region with boundary $\partial \mathcal{P}$ occupied by the shell in the reference placement, $Q_{R}(\mathbf{x}, t)$ is the referential mass (density) per unit volume of $\mathcal{P}, \eta(\mathbf{x}, t)$ and $\mathrm{r}(\mathbf{x}, t)$ are the 3D entropy and heat supply per unit mass of $\mathcal{P}, \Theta(\mathbf{x}, t)>0$ is the 3D absolute temperature, $\mathbf{q}(\mathbf{x}, t)$ is the heat flux vector through $\partial \mathcal{P}$ with $\mathbf{n}$ as the external unit normal, and $\mathbf{x}$ is the position vector of the place in $\mathcal{P}$ and on $\partial \mathcal{P}$.

In shell theory we usually parameterise points in $\mathcal{P}$ by $\mathbf{x} \quad \boldsymbol{x}+\xi \boldsymbol{\eta}$, where $\xi \in\left[h^{-}(\boldsymbol{x}), h^{+}(\boldsymbol{x})\right]$ is the distance along $\boldsymbol{\eta}$ from $M$ to $\mathbf{x} \in \mathcal{P}$, and $h \quad h^{-}+h^{+}$is the initial shell thickness.

In this paper we introduce after Murdoch (1976a) the mean referential temperature $\theta(\boldsymbol{x}, t)>0$ and the temperature deviation $\varphi(\boldsymbol{x}, t)$ by

$$
\frac{1}{\theta} \quad \frac{1}{2}\left(\frac{1}{\Theta_{+}}+\frac{1}{\Theta_{-}}\right), \quad \varphi \quad \frac{1}{h}\left(\frac{1}{\Theta_{-}} \frac{1}{\Theta_{+}}\right), \quad \frac{1}{\Theta_{+}} \quad \frac{1}{\theta} \quad \varphi \frac{h}{2}, \quad \frac{1}{\Theta_{-}} \quad \frac{1}{\theta}+\varphi \frac{h}{2}, \quad \Theta(\xi) \quad \frac{\theta}{1 \theta \varphi \xi},
$$

where $\Theta_{+}$are temperatures of the upper and lower shell faces $M^{ \pm}$taken to be equal to those prevailing in the adjoining external media. Then the direct through-the-thickness integration in (10) with (11) and (12) allows one to represent (10) in the form

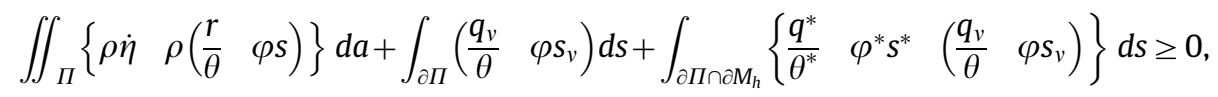

where

$$
\begin{array}{lll}
\rho \eta & \int_{-}^{+} \varrho_{R} \eta \mu d \xi, \quad \int_{-}^{+}(\cdot) \equiv \int_{-h}^{h^{+}}(\cdot), \\
\rho r & \int_{-}^{+} \varrho_{R} \mathrm{r} \mu d \xi \quad\left(\mathbf{q}_{+} \cdot \mathbf{n}^{+} \alpha^{+}+\mathbf{q}_{-} \cdot \mathbf{n}^{-} \alpha^{-}\right), \\
\rho s & \int_{-}^{+} \varrho_{R} \mathrm{r} \xi \mu d \xi & \frac{h}{2}\left(\mathbf{q}_{+} \cdot \mathbf{n}^{+} \alpha^{+} \quad \mathbf{q}_{-} \cdot \mathbf{n}^{-} \alpha^{-}\right), \\
q_{v} & \int_{-}^{+} \mathbf{q} \cdot \mathbf{n}^{*} \mu d \xi, \quad s_{v} \quad \int_{-}^{+} \mathbf{q} \cdot \mathbf{n}^{*} \xi \mu d \xi, \\
q^{*} & \int_{-}^{+} \mathbf{q}^{*} \cdot \mathbf{n}^{*} \mu d \xi, \quad s^{*} \quad \int_{-}^{+} \mathbf{q}^{*} \cdot \mathbf{n}^{*} \xi \mu d \xi,
\end{array}
$$

$\mathbf{q}^{-} \mathbf{q}\left(\boldsymbol{x} h^{-} \boldsymbol{\eta}(\boldsymbol{x})\right), \mathbf{q}^{+} \quad \mathbf{q}\left(\boldsymbol{x}+h^{+} \boldsymbol{\eta}(\boldsymbol{x})\right), \mathbf{q}^{*}(\mathbf{x})$ is the given heat flux vector through the lateral shell boundary surface $\partial \mathcal{P}^{*}, \theta^{*}$ and $\varphi^{*}$ are given functions along $\partial M_{h}$, and the geometric parameters $\mu, \alpha^{ \pm}, \mathbf{n}^{ \pm}, \mathbf{n}^{*}$ are given by Konopińska and Pietraszkiewicz (2007), Appendix (A.15)-(A.17).

In (14) we have introduced the referential resultant surface quantities: the internal entropy supply $\eta$, the heat supply $r$ and the extra heat supply $s$, all per unit surface mass of $M$, the heat fluxes $q_{v}$ and $q^{*}$ as well as the extra heat fluxes $s_{v}$ and $s^{*}$ trough the internal $\partial \Pi$ and external $\partial M_{h}$ edges, respectively. The fields $q_{v}$ and $s_{v}$ can be represented as $q_{v} \quad \boldsymbol{q} \cdot \boldsymbol{v}$ and $s_{v} \quad \boldsymbol{s} \cdot \boldsymbol{v}$, where $\boldsymbol{q}$ and $\boldsymbol{s}$ are the referential resultant heat flux and extra heat flux vectors.

Introducing (14) into (13) and applying the surface divergence theorem we obtain

$$
\begin{aligned}
& \iint_{\Pi \backslash C} \rho \dot{\eta} d a+\iint_{\Pi \cap \subset} V \llbracket \rho \eta \rrbracket d a \iint_{\Pi} \rho\left(\frac{r}{\theta} \quad \varphi s\right) d a+\iint_{\Pi \backslash C}\left(\frac{1}{\theta} \operatorname{Div} \boldsymbol{q} \frac{1}{\theta^{2}} \boldsymbol{q} \cdot \operatorname{Grad} \theta \quad \varphi \operatorname{Div} \boldsymbol{s}+\boldsymbol{s} \cdot \operatorname{Grad} \varphi\right) d a
\end{aligned}
$$

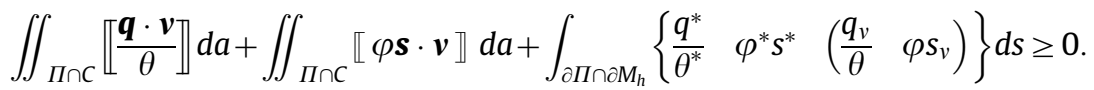

From (15) follow the local resultant 2D entropy inequalities of the non-linear theory of shells

$$
\begin{aligned}
& \rho \dot{\eta} \quad \rho\left(\frac{r}{\theta} \quad \varphi s\right)+\frac{1}{\theta} \operatorname{Div} \boldsymbol{q} \quad \varphi \operatorname{Div} \boldsymbol{s}+\boldsymbol{h} \cdot \boldsymbol{s} \quad \frac{1}{\theta^{2}} \boldsymbol{q} \cdot \mathbf{g} \geq 0 \text { in } \quad M \backslash C, \\
& V \llbracket \rho \eta \rrbracket \llbracket \frac{1}{\theta} \boldsymbol{q} \cdot \boldsymbol{v} \rrbracket+\llbracket \varphi \boldsymbol{s} \cdot \boldsymbol{v} \rrbracket \geq 0 \quad \text { along } C,
\end{aligned}
$$


$\frac{q^{*}}{\theta^{*}} \quad \varphi^{*} s^{*}\left(\begin{array}{ll}\frac{q_{v}}{\theta} & \varphi s_{v}\end{array}\right) \geq 0$ along $\partial M_{h}$

g $\operatorname{Grad} \theta, \quad \boldsymbol{h} \operatorname{Grad} \varphi, \quad \mathbf{g}, \boldsymbol{h} \in T_{x} M$.

Two surface fields $\theta$ and $\varphi$ used here require two respective dual fields, which are the resultant surface entropy $\eta$ and the surface entropy deviation $\chi$ (please do not identify this field with the deformation function used earlier). These fields appear naturally as multipliers of $\theta$ and $\varphi$ after the through-the-thickness integration of the 3D entropy distribution $\eta$ in

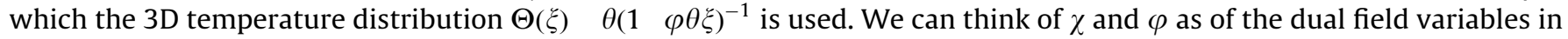
analogy to $\eta$ and $\theta$.

But from The 1st Law (8) we have

$\rho r \quad \operatorname{Div} \boldsymbol{q} \rho \dot{\varepsilon} \quad \mathbf{N} \bullet \dot{E} \quad \mathbf{M} \bullet \dot{\mathbf{K}}$,

where the referential shell stress and strain measures are defined by

$$
\mathbf{N} \quad \boldsymbol{Q}^{T} \boldsymbol{N}, \quad \mathbf{M} \quad \boldsymbol{Q}^{T} \boldsymbol{M}, \quad \mathbf{E} \quad \boldsymbol{Q}^{T} \boldsymbol{E}, \quad \mathbf{K} \quad \boldsymbol{Q}^{T} \boldsymbol{K} .
$$

Introducing the surface free energy density defined by

$$
\psi \quad \varepsilon \quad \theta \eta \quad \varphi \chi
$$

we can transform $(16)_{1}$ into

$$
\rho \delta \equiv \rho(\dot{\psi}+\dot{\theta} \eta+\varphi \dot{\chi}+\dot{\varphi} \chi)+\mathbf{N} \bullet \dot{\mathbf{E}}+\mathbf{M} \bullet \dot{\mathbf{K}} \quad \frac{1}{\theta} \boldsymbol{q} \cdot \mathbf{g}+\rho \theta \varphi s \quad \theta \varphi \text { Div } \boldsymbol{s} \quad \theta \boldsymbol{h} \cdot \boldsymbol{s} \geq 0 .
$$

In the balance laws (5),$(8)$ and in the inequality (20) valid in $M \backslash C$, the fields $\rho \boldsymbol{f}, \boldsymbol{c}, r, s$ are supposed to be given, the fields $\boldsymbol{u}, \boldsymbol{Q}, \theta, \varphi$ constitute the basic thermo-kinematic independent variables of the shell boundary-value problem, while the fields $\mathbf{N}, \mathbf{M}, \psi, \eta, \chi, \mathbf{q}, \boldsymbol{s}$ have to be specified by the constitutive equations. In what follows we discuss the constitutive equations for thermoelastic and thermoviscoelastic shells with the help of procedure worked out by Coleman and Noll (1963) in the 3D continuum thermodynamics.

\subsection{Thermoelastic shells}

The thermoelastic shell behaviour can be defined by the following constitutive equations:

$$
\begin{aligned}
& \psi \psi(\mathbf{E}, \mathbf{K}, \theta, \mathbf{g}, \varphi, \boldsymbol{h}), \\
& \eta \quad \eta(\mathbf{E}, \mathbf{K}, \theta, \mathbf{g}, \varphi, \boldsymbol{h}), \quad \chi \quad \chi(\mathbf{E}, \mathbf{K}, \theta, \mathbf{g}, \varphi, \boldsymbol{h}), \\
& \mathbf{N} \mathbf{N}(\mathbf{E}, \mathbf{K}, \theta, \mathbf{g}, \varphi, \boldsymbol{h}), \quad \mathbf{M} \quad \mathbf{M}(\mathbf{E}, \mathbf{K}, \theta, \mathbf{g}, \varphi, \boldsymbol{h}), \\
& \boldsymbol{q} \boldsymbol{q}(\mathbf{E}, \mathbf{K}, \theta, \mathbf{g}, \varphi, \boldsymbol{h}), \quad \boldsymbol{s} \quad \boldsymbol{s}(\mathbf{E}, \mathbf{K}, \theta, \mathbf{g}, \varphi, \boldsymbol{h}),
\end{aligned}
$$

where the constitutive functions and their values are denoted by the same symbols.

Then the local $2^{\text {nd }}$ Law (20) yields

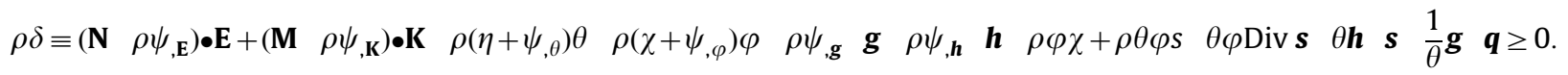

From (22) follows the inequality

$$
\begin{aligned}
& \rho \delta \equiv\left[\mathbf{N} \quad \rho(\psi+\varphi \chi)_{, \mathbf{E}}\right] \bullet \dot{\mathbf{E}}+\left[\begin{array}{lll}
\mathbf{M} & \rho(\psi+\varphi \chi)_{, \mathbf{K}}
\end{array}\right] \bullet \dot{\mathbf{K}} \quad \rho\left[\eta+(\psi+\varphi \chi)_{, \theta}\right] \dot{\theta} \quad \rho(\psi+\varphi \chi)_{, \varphi} \dot{\varphi} \quad \rho(\psi+\varphi \chi)_{\mathbf{g}} \cdot \dot{\boldsymbol{g}} \\
& \rho(\psi+\varphi \chi)_{, \boldsymbol{h}} \cdot \dot{\boldsymbol{h}}+\rho \theta \varphi s \quad \theta \varphi \operatorname{Div} \boldsymbol{s} \quad \theta \boldsymbol{h} \cdot \boldsymbol{s} \quad \frac{1}{\theta} \boldsymbol{g} \cdot \boldsymbol{q} \geq 0 .
\end{aligned}
$$

Let us recall the procedure of Coleman and Noll (1963) by which the entropy inequality could be used to deduce constitutive restrictions on a variety of materials. In this procedure it is assumed that time rates of arguments in the constitutive equations (21), i.e. $\dot{\mathbf{E}}, \dot{\mathbf{K}}$, etc., are all independent of each other. Hence, the inequality (23) should be satisfied for any constitutive Eqs. (21) and for any admissible values of the state variables $\mathbf{E}, \mathbf{K}, \theta, \varphi$ and their rates at any regular point of $M \backslash C$.

Let us consider the following family of deformations:

$$
\begin{aligned}
& \text { E } \quad \mathbf{E}_{0}+\left(\begin{array}{llll}
t & t_{0}
\end{array}\right) \mathbf{E}_{1}, \quad \mathbf{K} \quad \mathbf{K}_{0}+\left(\begin{array}{ll}
t & t_{0}
\end{array}\right) \mathbf{K}_{1}, \\
& \theta \quad \theta_{0}+\left(\begin{array}{ll}
t & t_{0}
\end{array}\right) \theta_{1}+\boldsymbol{g}_{0} \cdot\left(\begin{array}{ll}
\boldsymbol{x} & \boldsymbol{x}_{0}
\end{array}\right)+\boldsymbol{g}_{1} \cdot\left(\begin{array}{lll}
\boldsymbol{x} & \boldsymbol{x}_{0}
\end{array}\right)\left(\begin{array}{ll}
t & t_{0}
\end{array}\right), \\
& \varphi \quad \varphi_{0}+\left(\begin{array}{ll}
t & t_{0}
\end{array}\right) \varphi_{1}+\boldsymbol{h}_{0} \cdot\left(\begin{array}{ll}
\boldsymbol{x} & \boldsymbol{x}_{0}
\end{array}\right)+\boldsymbol{h}_{1} \cdot\left(\begin{array}{ll}
\boldsymbol{x} & \boldsymbol{x}_{0}
\end{array}\right)\left(\begin{array}{ll}
t & t_{0}
\end{array}\right),
\end{aligned}
$$


where $\mathbf{E}_{0}, \mathbf{E}_{1}, \mathbf{K}_{0}, K_{1}, \theta_{0}, \theta_{1}, \mathbf{g}_{0}, \mathbf{g}_{1}, \varphi_{0}, \varphi_{1}, \boldsymbol{h}_{0}$, and $\boldsymbol{h}_{1}$ are any constants, while $t_{0}$ and $\boldsymbol{x}_{0}$ are any time instant and any point on the shell surface. Substituting (24) into (23) and taking into account that at $t t_{0}, \boldsymbol{x} \boldsymbol{x}_{0}$,

$$
\begin{array}{cccccccccccc}
\mathbf{E} & \mathbf{E}_{0}, & \mathbf{K} & \mathbf{K}_{0}, & \theta & \theta_{0}, & \varphi & \varphi_{0}, & \mathbf{g} & \mathbf{g}_{0}, & \boldsymbol{h} & \boldsymbol{h}_{0}, \\
\dot{\mathbf{E}} & \mathbf{E}_{1}, & \dot{\mathbf{K}} & \mathbf{K}_{1}, & \dot{\theta} & \theta_{1}, & \dot{\varphi} & \varphi_{1}, & \dot{\mathbf{g}} & \mathbf{g}_{1}, & \boldsymbol{h} & \boldsymbol{h}_{1},
\end{array}
$$

we obtain the inequality

$$
\begin{aligned}
& \rho \delta \equiv\left[\mathbf{N} \quad \rho(\psi+\varphi \chi)_{, \mathbf{E}}\right] \bullet \mathbf{E}_{1}+\left[\begin{array}{ll}
\mathbf{M} & \left.\rho(\psi+\varphi \chi)_{, \mathbf{K}}\right] \bullet \mathbf{K}_{1} \quad \rho\left[\eta+(\psi+\varphi \chi)_{,}\right]
\end{array}\right] \theta_{1} \\
& \rho(\psi+\varphi \chi)_{, \varphi} \varphi_{1} \quad \rho(\psi+\varphi \chi)_{,} \cdot \mathbf{g}_{1} \quad \rho(\psi+\varphi \chi)_{, \boldsymbol{h}} \cdot \boldsymbol{h}_{1}+\rho \theta \varphi s \quad \theta \varphi \operatorname{Div} \boldsymbol{s} \quad \theta \boldsymbol{h} \cdot \boldsymbol{s} \quad \frac{1}{\theta} \mathbf{g} \cdot \boldsymbol{q} \geq 0 .
\end{aligned}
$$

Note that in (26), $\delta$ is the linear function with respect to $\mathbf{E}_{1}, \mathbf{K}_{1}, \theta_{1}, \varphi_{1}, \mathbf{g}_{1}$, and $\boldsymbol{h}_{1}$. Hence, to satisfy (26) we should have the relations

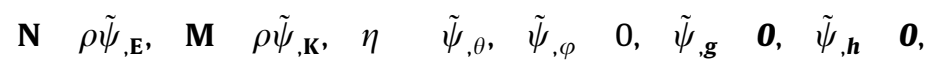

where $\tilde{\psi} \quad \tilde{\psi}(\mathbf{E}, \mathbf{K}, \theta) \equiv \psi+\varphi \chi$ does not depend on $\varphi, \mathbf{g}$ and $\boldsymbol{h}$, and the local $2^{\text {nd }}$ Law reduces to

$$
\theta \varphi[\rho s \quad \operatorname{Div} \boldsymbol{s}] \quad \theta \boldsymbol{h} \cdot \boldsymbol{s} \quad \frac{1}{\theta} \mathbf{g} \cdot \boldsymbol{q} \geq 0 .
$$

Assuming $\mathbf{g} \boldsymbol{0}$ and $\boldsymbol{h} \quad \boldsymbol{O}$ we find that the first group of terms in (28) has to be non-negative. Hence, to satisfy (28) we should have

$$
\theta \varphi[\rho s \quad \operatorname{Div} \boldsymbol{s}] \geq 0 .
$$

Note that the sign and value of $\varphi$ as well as the sign and value of Div $\boldsymbol{s}$ can be positive or negative, while $s$ is given function with a priory unknown sign and value. Hence, to satisfy (29) with $\theta>0$ we should have

$$
\rho s \quad \operatorname{Div} \boldsymbol{s} \equiv c \varphi,
$$

with $c \geq 0$. The quantity $c$ is functionally dependent on the state variables $\mathbf{E}, \mathbf{K}, \theta, \mathbf{g}, \varphi, \boldsymbol{h}$, in general. The simplest case is when $c$ const is assumed.

Let us note that for the entropy deviation $\chi$ we cannot find any restriction similar to that for $\eta$ in (27). Indeed, the relation

$$
(\psi+\varphi \chi)_{, \varphi} \quad 0
$$

following from $(27)_{4}$ yields

$$
\chi \quad\left(\begin{array}{ll}
\chi_{0} & \psi
\end{array}\right) / \varphi,
$$

where $\chi_{0} \quad \chi_{0}(\mathbf{E}, \mathbf{K}, \theta, \mathbf{g}, \boldsymbol{h})$ is an arbitrary function. Because $\psi \quad \psi(\mathbf{E}, \mathbf{K}, \theta), \chi$ depends linearly on $\varphi^{-1}$ and has a singularity when $\varphi \rightarrow 0$. Moreover, because $\chi_{0}$ is arbitrary, the constitutive relation for $\chi$ should be determined independently of $\psi$ taking into account the linear dependence of $\chi$ on $\varphi^{-1}$ alone. This dependence $\chi$ on $\varphi$ seems unwanted because the thermodynamic state with $\varphi \quad 0$ is physically reasonable but corresponds to the infinite value of the entropy deviation.

From (27) it also follows that $\mathbf{M}$ does not depend on $\varphi$. But from the elementary knowledge of beam and plate theories one knows that the case of non-zero temperature gradient leads to the bending of a beam or a plate, see for example Timoshenko and Woinowsky-Krieger (1985). This means that $\mathbf{M} \neq \mathbf{0}$ if $\varphi \neq 0$, in general. Hence, the latter property of the constitutive Eqs. (27) seems to be unsatisfactory as well. This unwanted consequence of the standard Coleman-Noll procedure was noted by Simmonds (1984) who proposed to treat $\dot{\varphi}$ as an internal variable.

Here we propose another solution how to avoid contradictions. The unsatisfactory behaviour of the above constitutive equations allows us to conclude that the independence of $\dot{\mathbf{E}}, \dot{\mathbf{K}}, \dot{\theta}$, and $\dot{\varphi}$ used above may not hold in the resultant $2 \mathrm{D}$ thermodynamics of shells. Thus, in the present paper we assume that $\dot{\mathbf{E}}, \dot{\mathbf{K}}, \dot{\theta}$, and $\dot{\varphi}$ are not independent. In other words, we do not consider all processes but restrict ourselves to such processes which are consistent with the 2D entropy inequality $(16)_{1}$. We can call such processes thermodynamically admissible.

Let us derive the governing equations of thermoelastic shells taking into account these constraints. Assuming that only $\dot{\mathbf{E}}, \dot{\mathbf{K}}$, and $\dot{\theta}$ are independent and considering the family

$$
\left.\begin{array}{lllllll}
\mathbf{E} & \mathbf{E}_{0}+(t & t_{0}
\end{array}\right) \mathbf{E}_{1}, \quad \mathbf{K} \quad \mathbf{K}_{0}+\left(\begin{array}{llll}
t & t_{0}
\end{array}\right) \mathbf{K}_{1}, \quad \theta \quad \theta_{0}+\left(\begin{array}{ll}
t & t_{0}
\end{array}\right) \theta_{1}+\mathbf{g}_{0} \cdot\left(\begin{array}{lll}
\boldsymbol{x} & \boldsymbol{x}_{0}
\end{array}\right)+\mathbf{g}_{1} \cdot\left(\begin{array}{lll}
\boldsymbol{x} & \boldsymbol{x}_{0}
\end{array}\right)\left(\begin{array}{ll}
t & t_{0}
\end{array}\right),
$$

we obtain from (22) that

$$
\psi \psi(\mathbf{E}, \mathbf{K}, \theta, \varphi, \boldsymbol{h}), \quad \mathbf{N} \quad \rho \psi_{, \mathbf{E}}, \quad \mathbf{M} \quad \rho \psi_{, \mathbf{K}}, \quad \eta \quad \psi_{, \theta},
$$

and (22) results in

$$
\rho \delta \equiv \rho\left(\chi+\psi_{, \varphi}\right) \dot{\varphi} \quad \rho \psi_{, \boldsymbol{h}} \cdot \dot{\boldsymbol{h}} \quad \rho \varphi \dot{\chi}+\rho \theta \varphi s \quad \theta \varphi \operatorname{Div} \boldsymbol{s} \quad \theta \boldsymbol{h} \cdot \boldsymbol{s} \quad \frac{1}{\theta} \boldsymbol{g} \cdot \boldsymbol{q} \geq 0 .
$$

Thus, $\varphi$ and $\chi$ should satisfy the inequality (33). 
Let us take the relation

$$
\chi \quad \psi_{, \varphi}
$$

as the constitutive equation for $\chi$, which is analogous to $(32)_{4}$, and assume the relation

$$
\psi_{, \boldsymbol{h}} \mathbf{0} .
$$

Note that with (35) the surface free energy density $\psi$ does not depend on $\boldsymbol{g}$ and $\boldsymbol{h}$, but depends on $\varphi$, and now $\mathbf{M}$ depends on $\varphi$, in general.

With (34) and (35) the local 2nd Law (33) reduces to

$$
\rho \varphi \dot{\chi}+\rho \theta \varphi s \quad \theta \varphi \operatorname{Div} \boldsymbol{s} \quad \theta \boldsymbol{h} \cdot \boldsymbol{s} \quad \frac{1}{\theta} \mathbf{g} \cdot \boldsymbol{q} \geq 0 .
$$

To satisfy (36) instead of (30), we should have the following relation:

$$
\rho \dot{\chi}+\theta \rho s \quad \theta \text { Divs } \quad c \varphi, \quad c \geq 0 .
$$

The reduced dissipation inequality (37), with $c \quad 0$ or when $\varphi \quad 0$ is assumed for a moment, becomes

$$
\theta \boldsymbol{h} \cdot \boldsymbol{s} \frac{1}{\theta} \mathbf{g} \cdot \boldsymbol{q} \geq 0,
$$

so that the sum of two last terms in (36) together have to be non-negative as well.

The simplest cases of the constitutive equations for $\boldsymbol{q}$ and $\boldsymbol{s}$ satisfying the reduced inequality (38) may be taken similar to the referential Fourier law in 3D continuum mechanics. These equations are

$$
\boldsymbol{q} \quad c_{\|} \mathbf{g}, \quad \boldsymbol{s} \quad c_{\perp} \boldsymbol{h},
$$

where $c_{\|}$is the positive heat conductivity of the shell in tangential direction and $c_{\perp}$ is the positive heat conductivity of the shell in the transverse normal direction.

For the thermoelastic shells the local energy balance Eq. (8) reduces to the form

$$
\rho(\theta \dot{\eta}+\varphi \dot{\chi}) \quad \rho r \quad \operatorname{Div} \boldsymbol{q} .
$$

Both relations (40) and (37) play the role of the thermoconductivity equations in the theory of thermoelastic shells. The two equations are necessary to determine two fields: the surface temperature $\theta$ and the surface temperature deviation $\varphi$. When $\boldsymbol{s} \mathbf{0}$, the Eq. (37) contains as the special case the equation for temperature deviation established for the thermoelastic beams by Simmonds (2005).

\subsection{Thermoviscoelastic shells of differential type}

The discussion given in Section 5.1 above may be extended to the case of inelastic shell behaviour. Let us consider the more general case of thermoviscoelastic shells of the differential type defined by the following constitutive equations:

$$
\{\psi, \eta, \chi, \mathbf{N}, \mathbf{M}, \boldsymbol{q}, \mathbf{s}\} \quad\{\psi, \eta, \chi, \mathbf{N}, \mathbf{M}, \boldsymbol{q}, \boldsymbol{s}\}(\mathbf{E}, \mathbf{K}, \dot{\mathbf{E}}, \dot{\mathbf{K}}, \theta, \mathbf{g}, \dot{\theta}, \dot{\mathbf{g}}, \varphi, \boldsymbol{h}, \dot{\varphi}, \dot{\boldsymbol{h}}) .
$$

Here we additionally take into account dependence of the constitutive equations on the first derivatives with respect to time-like parameter of thermo-kinematic variables and their spatial gradients. Eqs. (41) are analogous to the 3D constitutive equations of the differential type of the complexity 1 (the Kelvin-Voigt type model), which were discussed in Truesdell $(1984,1991)$.

Let the equilibrium and dissipative parts of the surface stress measures $\mathbf{N}$ and $\mathbf{M}$ be additionally decomposed according to

$$
\begin{array}{llll}
\mathbf{N} & \mathbf{N}_{E}+\mathbf{N}_{D}, \quad \mathbf{M} \quad \mathbf{M}_{E}+\mathbf{M}_{D}, \\
\mathbf{N}_{E} & \mathbf{N}_{E}(\mathbf{E}, \mathbf{K}, \theta, \mathbf{g}, \varphi, \boldsymbol{h}), \quad \mathbf{M}_{E} & \mathbf{M}_{E}(\mathbf{E}, \mathbf{K}, \theta, \mathbf{g}, \varphi, \boldsymbol{h}), \\
\mathbf{N}_{D}(\mathbf{E}, \mathbf{K}, \mathbf{0}, \mathbf{0}, \theta, \mathbf{g}, \mathbf{0}, \mathbf{0}, \varphi, \boldsymbol{h}, \mathbf{0}, \mathbf{0}) & \mathbf{0}, \quad \mathbf{M}_{D}(\mathbf{E}, \mathbf{K}, \mathbf{0}, \mathbf{0}, \theta, \mathbf{g}, 0, \mathbf{0}, \varphi, \boldsymbol{h}, 0, \mathbf{0}) & \mathbf{0} .
\end{array}
$$

Substituting (41) and (42) into (20) we obtain

$$
\begin{aligned}
& \begin{array}{llllllllllll}
\rho & \left(\mathbf{N}_{E}\right. & \rho \psi_{, \mathbf{E}} & \mathbf{E}+\left(\mathbf{M}_{E}\right. & \left.\rho \psi_{, \mathbf{K}}\right) & \mathbf{K} & \rho\left(\eta+\psi_{, \theta}\right) & \theta & \rho \psi_{, \dot{\mathbf{E}}} & \ddot{\mathbf{E}} & \rho \psi_{, \dot{\mathbf{K}}} & \ddot{\mathbf{K}}
\end{array} \\
& \rho \psi_{, \mathbf{g}} \cdot \dot{\mathbf{g}} \quad \rho \psi_{, \dot{\mathbf{g}}} \cdot \ddot{\mathbf{g}} \rho\left(\chi+\psi_{, \varphi}\right) \dot{\varphi} \quad \rho \psi_{, \boldsymbol{h}} \cdot \dot{\boldsymbol{h}} \\
& \rho \psi_{, \dot{\varphi}} \ddot{\varphi} \quad \rho \psi_{, \dot{\boldsymbol{h}}} \cdot \ddot{\boldsymbol{h}}+\mathbf{N}_{D} \cdot \dot{\mathbf{E}}+\mathbf{M}_{D} \cdot \dot{\mathbf{K}} \quad \frac{1}{\theta} \mathbf{g} \cdot \boldsymbol{q} \quad \theta \boldsymbol{h} \cdot \boldsymbol{s} \quad \rho \varphi \dot{\chi}+\rho \theta \varphi s \quad \varphi \theta \operatorname{Div} \boldsymbol{s} \geq 0 .
\end{aligned}
$$

The analysis of (43) similar to that in Section 5.1 leads to the relations

$$
\begin{aligned}
& \psi \psi(\mathbf{E}, \mathbf{K}, \theta, \varphi), \\
& \begin{array}{llllllll}
\mathbf{N}_{E} & \rho \psi_{, \mathbf{E}}, & \mathbf{M}_{E} & \rho \psi_{, \mathbf{K}}, & \eta & \psi_{, \theta}, & \chi & \psi_{, \varphi},
\end{array} \\
& \rho \dot{\chi}+\rho \theta s \quad \theta \operatorname{Div} \boldsymbol{s} \quad c \varphi, \quad c \geq 0,
\end{aligned}
$$




$$
\mathbf{N}_{D} \bullet \dot{\mathbf{E}}+\mathbf{M}_{D} \cdot \dot{\mathbf{K}} \quad \frac{1}{\theta} \boldsymbol{g} \cdot \boldsymbol{q} \quad \theta \boldsymbol{h} \cdot \boldsymbol{s} \geq 0
$$

Let us note that here the surface free energy density $\psi$ and the equilibrium surface stress measures $\mathbf{N}_{E}, \mathbf{M}_{E}$ are the same as in the case of thermoelastic shells, while $\mathbf{N}_{D}, \mathbf{M}_{D}, \boldsymbol{q}$, and $\boldsymbol{s}$ may depend on the full list of arguments including the temperature deviation $\varphi$, its surface gradient and their time-like derivatives.

\section{Thermodynamic continuity condition}

Let us discuss the relations (4), (6), (9) and $(16)_{2}$ for jumps of various fields at $C$. We remind that these relations should be satisfied for arbitrary shells, also elastic, thermoelastic and thermoviscoelastic. This is so because these relations either represent continuity as (4), or the balance equations of some fields at the singular curve $C$ quasistatically moving on the base surface $M$. Additionally, we assume that the surface temperature field $\theta$ and its deviation $\varphi$ are continuous on the whole $M$, that is

$$
\llbracket \theta \rrbracket \quad 0, \quad \llbracket \varphi \rrbracket \quad 0 \text { along } C .
$$

The second thermoconductivity Eq. (37) leads to the relation

$$
V \frac{1}{\theta} \llbracket \rho \chi \rrbracket \llbracket \boldsymbol{s} \cdot \boldsymbol{v} \rrbracket \quad 0 \text { along } C .
$$

In the derivation of this relation one uses the classical technique of the theory of partial differential equations, see for example Courant and Hilbert (1989), Truesdell (1966) or Maugin (1998) in the case of PT. Formally, the derivation procedure reduces to exchange of the differential operators $\operatorname{Div}(\cdots)$ and $(\cdots)$ by the algebraic operators $(\cdots) \cdot v$ and $V(\cdots)$, respectively.

Eliminating $\llbracket \boldsymbol{q} \cdot \boldsymbol{v} \rrbracket$ from (9) and (16) $)_{2}$, we have

$$
V \llbracket \rho(\theta \eta \quad \varepsilon) \rrbracket \llbracket \boldsymbol{n}_{v} \cdot \boldsymbol{v} \rrbracket \llbracket \boldsymbol{m}_{v} \cdot \boldsymbol{\omega} \rrbracket+\llbracket \theta \varphi \boldsymbol{s} \cdot \boldsymbol{v} \rrbracket \geq 0 .
$$

Substituting (19) into this inequality, we derive that

$$
V \llbracket \rho \psi \rrbracket \llbracket \boldsymbol{n}_{v} \cdot \boldsymbol{v} \rrbracket \llbracket \boldsymbol{m}_{v} \cdot \boldsymbol{\omega} \rrbracket \quad V \llbracket \rho \varphi \chi \rrbracket+\llbracket \theta \varphi \boldsymbol{s} \cdot \boldsymbol{v} \rrbracket \geq 0 .
$$

Using (45) and (46), the following relation holds along $C$ :

$$
V \llbracket \rho \psi \rrbracket \llbracket \boldsymbol{n}_{v} \cdot \boldsymbol{v} \rrbracket \llbracket \boldsymbol{m}_{v} \cdot \boldsymbol{\omega} \rrbracket \equiv \theta \delta_{\mathrm{C}}^{2} \geq 0,
$$

where $\delta_{C}^{2} \geq 0$ denotes the surface entropy production at $C$.

Using the identities

$$
\llbracket \boldsymbol{n}_{v} \cdot \boldsymbol{v} \rrbracket \quad\left\langle\boldsymbol{n}_{v}\right\rangle \cdot \llbracket \boldsymbol{v} \rrbracket+\llbracket \boldsymbol{n}_{v} \rrbracket \cdot\langle\boldsymbol{v}\rangle, \quad \llbracket \boldsymbol{m}_{v} \cdot \boldsymbol{\omega} \rrbracket \quad\left\langle\boldsymbol{m}_{v}\right\rangle \cdot \llbracket \boldsymbol{\omega} \rrbracket+\llbracket \boldsymbol{m}_{v} \rrbracket \cdot\langle\boldsymbol{\omega}\rangle,
$$

where $\langle\ldots\rangle \frac{1}{2}\left[(\ldots)_{A}+(\ldots)_{B}\right]$ is the mean value at $C$, and taking into account the static balance Eqs. (6) we obtain the relation

$$
V \llbracket \rho \psi \rrbracket\left\langle\boldsymbol{n}_{v}\right\rangle \cdot \llbracket \boldsymbol{v} \rrbracket\left\langle\boldsymbol{m}_{v}\right\rangle \cdot \llbracket \boldsymbol{\omega} \rrbracket \quad \theta \delta_{C}^{2} \text { along } C,
$$

or

$$
V \llbracket \rho \psi \rrbracket \boldsymbol{v} \cdot \boldsymbol{N}^{T} \llbracket \boldsymbol{v} \rrbracket \boldsymbol{v} \cdot \boldsymbol{M}^{T} \llbracket \boldsymbol{\omega} \rrbracket \quad \theta \delta_{C}^{2} \quad \text { along } C .
$$

For the coherent phase interface

$$
\theta \delta_{C}^{2} \quad V\left\{\llbracket \rho \psi \rrbracket \boldsymbol{v} \cdot \boldsymbol{N}^{T} \llbracket \boldsymbol{F v} \rrbracket \boldsymbol{v} \cdot \boldsymbol{M}^{T} \llbracket \boldsymbol{K} \boldsymbol{v} \rrbracket\right\} \text { along } C,
$$

while for the phase interface incoherent in rotations

$$
\theta \delta_{C}^{2} \quad V\left\{\llbracket \rho \psi \rrbracket \boldsymbol{v} \cdot \boldsymbol{N}^{T} \llbracket \boldsymbol{F} \boldsymbol{v} \rrbracket\right\} \text { along } C .
$$

The entropy production $\theta \delta_{C}^{2}$ remains always non-negative for all thermomechanical processes. This allows us to postulate the kinetic equation, describing motion of the phase interface for all quasistatic processes, in the form

$$
V \quad \mathcal{F}(\boldsymbol{v} \cdot \llbracket \boldsymbol{C} \rrbracket v),
$$

where $\mathcal{F}$ is the non-negative definite kinetic function depending on the jump of $\boldsymbol{C}$ at $C$, i.e. $\mathcal{F}(\varsigma) \geq 0$ for $\varsigma>0$, where

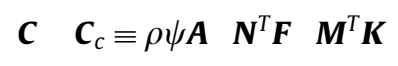

for the coherent interface,

$$
\text { C } \quad \boldsymbol{C}_{i} \equiv \rho \psi \boldsymbol{A} \quad \mathbf{N}^{T} \boldsymbol{F}
$$

for the one incoherent in rotations, $\boldsymbol{A} \mathbf{1} \boldsymbol{\eta} \otimes \boldsymbol{\eta}$, and $\mathbf{1}$ is the 3D unit tensor.

Tensors $\boldsymbol{C}_{c}$ and $\boldsymbol{C}_{i}$ in the non-linear shell theory play the role of the Eshelby tensors or the energy-momentum tensors known in 3D continuum mechanics. Let us note that these tensors have various applications not only in the theory of PT 
but also in the configurational mechanics (Berezovski et al., 2008; Gurtin, 2000; Maugin, 1993; Kienzler and Herrman, 2000). In particular, in Kienzler and Herrman (2000) properties of the Eshelby tensor were used to formulate the conservation laws and the path-independent integrals in the linear theory of plates and shells.

After Abeyaratne and Knowles (2006), Berezovski et al. (2008), and Eremeyev and Pietraszkiewicz (2010) we assume $\mathcal{F}(\varsigma)$ in the form

$$
\mathcal{F}(\varsigma)\left\{\begin{array}{lc}
\frac{k\left(\varsigma \varsigma_{0}\right)}{1+\xi\left(\varsigma \varsigma_{0}\right)}, & \varsigma \geq \varsigma_{0}, \\
0, & \varsigma_{0}<\varsigma<\varsigma_{0}, \\
\frac{k\left(\varsigma+\varsigma_{0}\right)}{1 \xi\left(\varsigma+\varsigma_{0}\right)}, & \varsigma \leq \varsigma_{0} .
\end{array}\right.
$$

Here $\varsigma_{0}$ describes the effects associated with nucleation of the new phase and action of the surface tension, see Abeyaratne and Knowles (2006), $\xi$ is a parameter describing the limit value of the phase transition velocity (Berezovski et al., 2008), and $k$ is a positive kinetic factor.

Summarising, in the case of finite deformations the thermomechanic BVP for the shell undergoing phase transition consists of the equilibrium Eqs. (5) $)_{1}$ supplemented by the appropriate static and kinematic boundary conditions for $\boldsymbol{N}, \boldsymbol{M}, \boldsymbol{u}$, and $\boldsymbol{Q}$, the energy balance Eq. (8) with appropriate boundary conditions for $\theta$ and $\varphi$, the surface entropy inequality $(16)_{2}$, as well as the balance Eqs. (4), (6), (45) and (50) along the interface $C$, all supplemented with the constitutive equations derived in Sections 5.1 or 5.2. The Eq. (50) is used to find position of the curvilinear interface $C$ in its quasistatic motion.

\section{Stretching and bending of the circular plate undergoing PT}

Let us consider the axisymmetric quasistatic deformation of thermoelastic circular plate with simply supported external boundary under the action of tensile forces $F$ and distributed transverse loads $q$, see Fig. 1 . We assume that strains are small everywhere and that the deformation process is isothermal with the constant surface temperature $\theta$ and the constant surface temperature deviation $\varphi$. When deformation is small one can essentially simplify expressions for the strain measures (3). In such a case $\boldsymbol{\varepsilon}_{\alpha}$ and $\boldsymbol{\varkappa}_{\alpha}$ are given by, see Chróścielewski et al. (2004),

$$
\begin{array}{lllll}
\boldsymbol{\varepsilon}_{\alpha} & \boldsymbol{u}_{, \alpha} & \boldsymbol{\vartheta} \times \boldsymbol{x}_{, \alpha}, \quad \boldsymbol{x}_{\alpha} & \boldsymbol{\vartheta}_{, \alpha},
\end{array}
$$

where $\boldsymbol{\vartheta}$ is the infinitesimal rotation vector such that $\boldsymbol{Q} \approx \mathbf{1}+\boldsymbol{9} \times \mathbf{1}$ if $\|\boldsymbol{\vartheta}\| \ll 1$. Hence, in the case of small strains the strain measures are given by

\section{E Grad $\boldsymbol{u} \boldsymbol{\vartheta} \times \mathbf{1}, \quad \mathbf{K}$ Grad $\boldsymbol{\vartheta}$.}

Note that in such a case we approximately have $\mathbf{N} \cong \mathbf{N}, \boldsymbol{M} \cong \mathbf{M}, \boldsymbol{E} \cong \mathbf{E}, \mathbf{K} \cong \mathbf{K}$.

We assume that the plate consists of two phases, say $A$ and $B$. All quantities related to these phases we denote by using indices $A$ and $B$, respectively.

Let us consider the following constitutive equations for the phases $A, B$ :

$$
\begin{aligned}
2 \rho \psi^{A, B} & \alpha_{1}^{A, B} \operatorname{tr}^{2} \tilde{\mathbf{E}}_{\|}+\alpha_{2}^{A, B} \operatorname{tr} \tilde{\mathbf{E}}_{\|}^{2}+\alpha_{3}^{A, B} \operatorname{tr}\left(\tilde{\mathbf{E}}_{\|}^{T} \tilde{\mathbf{E}}_{\|}\right)+\alpha_{4}^{A, B} \boldsymbol{\eta} \cdot \mathbf{E} \mathbf{E}^{T} \boldsymbol{\eta}+\beta_{1}^{A, B} \operatorname{tr}^{2} \tilde{\mathbf{K}}_{\|}+\beta_{2}^{A, B} \operatorname{tr} \tilde{\mathbf{K}}_{\|}^{2}+\beta_{3}^{A, B} \operatorname{tr}\left(\tilde{\mathbf{K}}_{\|}^{T} \tilde{\mathbf{K}}_{\|}\right)+\beta_{4}^{A, B} \boldsymbol{\eta} \cdot \mathbf{K K}^{T} \boldsymbol{\eta} \\
& +\alpha\left(\theta \quad \theta_{0}\right) \operatorname{tr} \mathbf{E}+\beta \varphi \operatorname{tr}(\boldsymbol{\eta} \times \mathbf{K})+2 \rho \psi_{0}^{A, B}(\theta, \varphi) .
\end{aligned}
$$

Here $\alpha_{k}, \beta_{k}$ are the tangential stiffness and bending stiffness parameters, $k \quad 1,2,3,4, \alpha$ and $\beta$ are the coefficients describing the coupling between temperature and stress measures, $\tilde{\mathbf{E}} \mathbf{E} \mathbf{E}_{p}^{A, B}, \tilde{\mathbf{K}} \mathbf{K} \quad \mathbf{K}_{p}^{A, B}$, where $\mathbf{E}_{p}^{A, B}$ and $\mathbf{K}_{p}^{A, B}$ are phase transformation strains, $\mathbf{E}_{\|} \quad \mathbf{A E} \in T_{x} M \otimes T_{x} M, \mathbf{K}_{\|} \quad \mathbf{A K} \in T_{x} M \otimes T_{x} M, \theta_{0}$ is the reference mid-surface temperature, and $\psi_{0}$ is the surface free energy density when strains are zero. We assume that $\mathbf{E}_{p}^{A, B} \quad \varepsilon_{p}^{A, B} \mathbf{A}, \mathbf{K}_{p}^{A, B} \quad \kappa_{p}^{A, B} \boldsymbol{\eta} \times \mathbf{A}$ with $\varepsilon_{p}^{B} \quad 0, \kappa_{p}^{B} \quad 0$. Such phase transformation strain measures correspond to an isotropic extension and bending of the material surface under the phase transition. The function (54) generates the following constitutive equations for the isotropic thermoelastic shell:

$$
\begin{array}{ll}
\mathbf{N} & \alpha_{1} \boldsymbol{A} \operatorname{tr} \tilde{\mathbf{E}}_{\|}+\alpha_{2} \tilde{\mathbf{E}}_{\|}^{T}+\alpha_{3} \tilde{\mathbf{E}}_{\|}+\alpha_{4} \boldsymbol{\eta} \otimes \mathbf{E}^{T} \boldsymbol{\eta}+\alpha\left(\theta \quad \theta_{0}\right) \mathbf{A}, \\
\mathbf{M} & \beta_{1} \boldsymbol{A} \operatorname{tr} \tilde{\mathbf{K}}_{\|}+\beta_{2} \tilde{\mathbf{K}}_{\|}^{T}+\beta_{3} \tilde{\mathbf{K}}_{\|}+\beta_{4} \boldsymbol{\eta} \otimes \mathbf{K}^{T} \boldsymbol{\eta}+\beta \varphi \boldsymbol{\eta} \times \mathbf{A},
\end{array}
$$

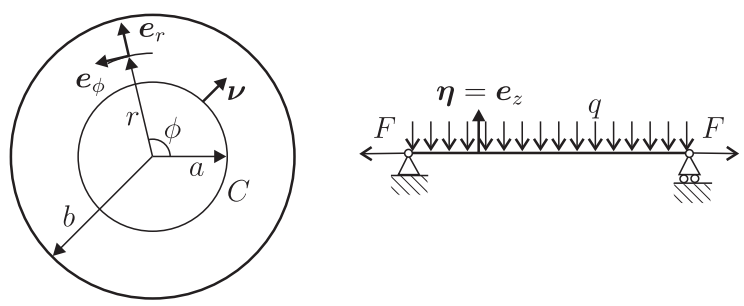

Fig. 1. Bending and tension of a two phase circular plate. 
In Chróścielewski et al. (2004) the following relations for the elastic moduli appearing in (54) and (55) were used:

$$
\begin{aligned}
& \alpha_{1} \quad C v, \quad \alpha_{2} \quad 0, \quad \alpha_{3} \quad C(1 \quad v), \quad \alpha_{4} \quad \alpha_{s} C\left(\begin{array}{lll}
1 & v
\end{array}\right), \\
& \beta_{1} \quad D v, \quad \beta_{2} \quad 0, \quad \beta_{3} \quad D(1 \quad v), \quad \beta_{4} \quad \alpha_{t} D(1 \quad v), \\
& \text { C } \frac{E h}{1 v^{2}}, \quad D \quad \frac{E h^{3}}{12\left(1 v^{2}\right)},
\end{aligned}
$$

where $E$ and $v$ are the Young modulus and the Poisson ratio of the bulk material, respectively, $\alpha_{s}$ and $\alpha_{t}$ are dimensionless shear correction factors, while $h$ is the shell thickness.

Let us consider the axisymmetric deformation of the plate described by

$$
\begin{array}{llll}
\boldsymbol{u} & u(r) \boldsymbol{e}_{r}+w(r) \boldsymbol{e}_{z}, \quad \vartheta \quad \vartheta(r) \boldsymbol{e}_{\phi},
\end{array}
$$

where $r, \phi$ are cylindrical coordinates, and $\boldsymbol{e}_{r}, \boldsymbol{e}_{\phi}, \boldsymbol{e}_{z}$ are the mid-surface base vectors. We initially assume existence of one phase interface $C$ to be a circle with an unknown radius $a$. Thus, the plate consists of two phases, say $A$ and $B$, separated by the circle $C$.

Using (53) and (57) we obtain

$$
\mathbf{E} \quad u^{\prime} \boldsymbol{e}_{r} \otimes \boldsymbol{e}_{r}+\frac{u}{r} \boldsymbol{e}_{\phi} \otimes \boldsymbol{e}_{\phi}+\left(\begin{array}{llll}
w^{\prime} & \vartheta
\end{array}\right) \boldsymbol{e}_{z} \otimes \boldsymbol{e}_{r}, \quad \mathbf{K} \quad \vartheta^{\prime} \boldsymbol{e}_{\phi} \otimes \boldsymbol{e}_{r} \quad \frac{\vartheta}{r} \boldsymbol{e}_{r} \otimes \boldsymbol{e}_{\phi},
$$

where $(. . .)^{\prime}$ denotes the derivative with respect to $r$. The stress measures take the form

$$
\mathbf{N} \quad N_{r r} \boldsymbol{e}_{r} \otimes \boldsymbol{e}_{r}+N_{\phi \phi} \boldsymbol{e}_{\phi} \otimes \boldsymbol{e}_{\phi}+N_{z r} \boldsymbol{e}_{z} \otimes \boldsymbol{e}_{r}, \quad \mathbf{M} \quad M_{\phi r} \boldsymbol{e}_{\phi} \otimes \boldsymbol{e}_{r}+M_{r \phi} \boldsymbol{e}_{r} \otimes \boldsymbol{e}_{\phi} .
$$

From (55) and (58) it follows that

$$
\begin{aligned}
& N_{r r} \quad \alpha_{1}\left(u^{\prime}+\frac{u}{r}\right)+\alpha_{3} u^{\prime} \quad\left(\begin{array}{lll}
\left.2 \alpha_{1}+\alpha_{3}\right) \varepsilon_{p}+\alpha(\theta & \theta_{0}
\end{array}\right), \\
& N_{\phi \phi} \quad \alpha_{1}\left(u^{\prime}+\frac{u}{r}\right)+\alpha_{3} \frac{u}{r} \quad\left(2 \alpha_{1}+\alpha_{3}\right) \varepsilon_{p}+\alpha\left(\begin{array}{ll}
\theta & \theta_{0}
\end{array}\right), \\
& N_{z r} \quad \alpha_{4}\left(w^{\prime} \quad \vartheta\right), \quad M_{\phi r} \quad \beta_{3} \vartheta^{\prime}+\beta_{3} \kappa_{p} \quad \beta \varphi, \\
& M_{r \phi} \quad \beta_{3} \frac{\vartheta}{r}+\beta_{3} \kappa_{p}+\beta \varphi .
\end{aligned}
$$

The equilibrium Eqs. (5) reduce here to three ordinary differential equations

$$
\begin{aligned}
& N_{r r}^{\prime}+\frac{1}{r}\left(\begin{array}{llll}
N_{r r} & \left.N_{\phi \phi}\right)+f \quad 0, & N_{z r}^{\prime}+\frac{1}{r} N_{z r}+q & 0, \\
M_{\phi r}^{\prime}+\frac{1}{r}\left(M_{\phi r}+M_{r \phi}\right)+c_{\phi} & 0,
\end{array}\right.
\end{aligned}
$$

where $f \quad \boldsymbol{f} \cdot \boldsymbol{e}_{r}, q \quad \boldsymbol{f} \cdot \boldsymbol{e}_{z}, c_{\phi} \quad \boldsymbol{c} \cdot \boldsymbol{e}_{\phi} \cdot N_{z r}$ can be found immediately under the assumption of constant values of the function $q$ and is given by the relation

$$
N_{z r} \quad \frac{q}{2 r}+\frac{c_{1}}{r}
$$

where $c_{1}$ is an integration constant.

Substituting (60) into (61) we obtain three 2 nd-order ODE for $u, w$, and $\vartheta$. In the case of constant values of the functions $f, q$, and $c_{\phi}$, the general solution of this system is given by

$$
\begin{array}{ll}
w & w_{0}+\frac{c_{1} r^{2}}{2}+c_{2} \ln r \frac{c_{\phi} r^{3}}{9 \beta_{3}} \frac{q r^{4}}{64 \beta_{3}} \frac{q r}{2 \alpha_{4}}, \\
u & d_{1} r+\frac{d_{2}}{r} \frac{f r^{2}}{3\left(\alpha_{1}+\alpha_{3}\right)}, \\
\vartheta & c_{1} r+\frac{c_{2}}{r} \quad \frac{c_{\phi} r^{2}}{3 \beta_{3}} \frac{q r^{3}}{16 \beta_{3}},
\end{array}
$$

where $c_{1}, c_{2}, d_{1}, d_{2}$, and $w_{0}$ are integration constants. Note that the assumed constant values of $\theta$ and $\varphi$ as well as dependence of $\mathbf{N}$ and $\mathbf{M}$ on $\varepsilon_{p}$ and $\kappa_{p}$ do not influence the form of solution (62), but have to be taken into account when one determines the integration constants from boundary conditions. 
The boundary conditions for the plate are given by the relations

$$
N_{r r} \quad F, \quad w \quad 0, \quad M_{\phi r} \quad 0
$$

at the external boundary $r \quad b$ of the plate, and

$$
\llbracket N_{r r} \rrbracket \quad \llbracket N_{z r} \rrbracket \quad 0, \quad \llbracket M_{\phi r} \rrbracket \quad 0, \quad \llbracket u \rrbracket \quad \llbracket w \rrbracket \quad 0, \quad \llbracket \vartheta \rrbracket \quad 0
$$

at the coherent phase interface $r$ a, or

$$
\llbracket N_{r r} \rrbracket \quad \llbracket N_{z r} \rrbracket \quad 0, \quad M_{\phi r} \quad 0, \quad \llbracket u \rrbracket \quad \llbracket w \rrbracket \quad 0
$$

at the phase interface $r$ a incoherent in rotations.

For the assumed loading we have $f \quad 0$ and $c_{\phi} \quad 0$.

The kinetic Eq. (50) takes the form

$$
\frac{d a}{d t} \quad \mathcal{F}(\varsigma) \text { at } r \quad a,
$$

where $\varsigma$ is given by

$$
\varsigma \quad \llbracket \rho \psi \quad N_{r r} u^{\prime} \quad N_{z r} w^{\prime} \quad M_{\phi r} \vartheta^{\prime} \rrbracket
$$

for the coherent phase interface, and

$$
\varsigma \quad \llbracket \rho \psi \quad N_{r r} u^{\prime} \quad N_{z r} w^{\prime} \rrbracket
$$

for the interface incoherent in rotations.

After calculating the integration constants from the boundary conditions (63), (64) or (65), Eq. (66) becomes the ODE with respect to $a$,

$$
\frac{d a}{d t} \hat{\mathcal{F}}(a ; F, q, \ldots)
$$

where $\hat{\mathcal{F}}(a ; F, q, \ldots)$ is the value of $\mathcal{F}(\varsigma)$ after substitution of the integration constants into $\varsigma$.

\subsection{Stretching}

Let us consider the simplest case when $\theta \quad \theta_{0}, \varphi \quad 0, q \quad 0$, and $\kappa_{p} \quad 0$. In this case one has the plane stress state with

$$
\begin{array}{llllllllllll}
w & 0, & \vartheta & 0, & u & d_{1} r+\frac{d_{2}}{r}, & N_{z r} & 0, & M_{r \phi} & M_{\phi r} & 0 .
\end{array}
$$

There is no difference between the coherent phase interface and the one incoherent in rotations, and $\varsigma \quad \llbracket \rho \psi \quad N_{r r} u^{\prime} \rrbracket$.

For the sake of simplicity let us assume that both phases have the same elastic moduli, i.e. $E^{A} E^{B}, v^{A} v^{B}$, and differ only by the phase transformation strain $\varepsilon^{A}$ and values of the surface free energy densities at zero strains, so that $\delta \equiv \rho \psi_{0}^{B} \quad \rho \psi_{0}^{A} \neq 0$.

Let us begin with the thermodynamic equilibrium. There are at least two solutions with one phase interface. Note that non-uniqueness of solutions of the boundary-value problems for elastic bodies undergoing phase transformations is a standard situation, see e.g. Eremeyev and Zubov (1991), Freidin et al. (2006), and Yeremeyev et al. (2007) for the 3D case.

We assume that at the initial moment the plate consists of one phase, say the phase $A$. The first solution describes the case when the new phase $B$ nucleates in the centre of the plate, i.e. at the point $r \quad a$. At the initial moment $F \quad 0$, and the plate consists of one phase $A$ and $a \quad 0$. When $F \quad F_{1}^{*}$ the new phase nucleates in the centre of the plate, and the interface radius $a$ increases from 0 to $b$ when $F$ attains the value $F_{2}^{*}$. If $F>F_{2}^{*}$ then the plate consists entirely of the phase $B$. Dependence of $a$ on $F$ is given in Fig. 2 as the solid curve.

The second solution describes the case when the phase $B$ appears at the plate boundary. If $F \quad F_{1}^{*}$ and the new phase appears at $r \quad b$, then $a$ decreases from $b$ to 0 when $F_{1}^{*}<F<F_{2}^{*}$, see Fig. 2 (dashed curve), and then the plate consists again entirely of the phase $B$. If the same elastic moduli of material phases are assumed, these two-phase solutions exist on the

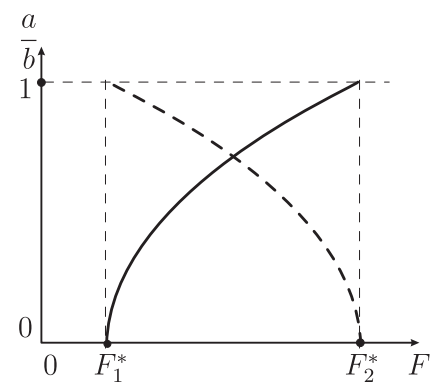

Fig. 2. Dependence of the phase interface radius $a$ on the external loads $F$. The solid curve describes the nucleation of the new phase $B$ in the centre of the plate, while the dashed curve concerns the creation of the new phase at the plate boundary. 


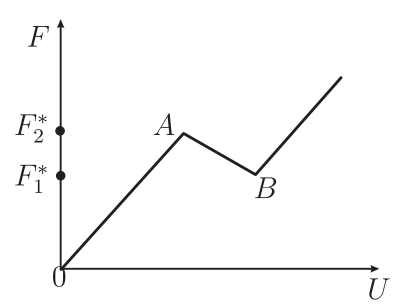

Fig. 3. Equilibrium $F \quad U$ diagram for the two phase plate.

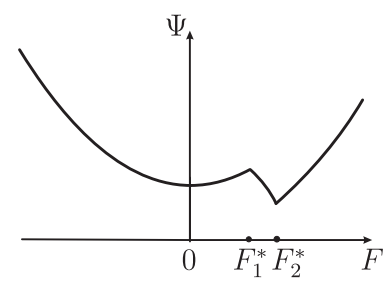

Fig. 4. Dependence of $\Psi$ on the external loads $F$.

same interval of $F$ and are symmetric under mirror reflection on the line $F \quad\left(F_{1}^{*}+F_{2}^{*}\right) / 2$. In the general case of different elastic moduli this symmetry is violated. The loading diagram for the plate is presented in Fig. 3, where $U \quad u(b) / b$ is the dimensionless translation of the plate boundary. The diagram consists of three parts. The line $A B$ relates to the two-phase state and corresponds the Maxwell line (Abeyaratne and Knowles, 2006). In contrast to the solution given in Eremeyev and Pietraszkiewicz (2009), where the two-phase state is described by the horizontal line, here we have the decreasing part of the diagram. This is analogous to the loading diagram for the two-phase elastic sphere (Eremeyev and Zubov, 1991; Freidin et al., 2006; Yeremeyev et al., 2007). The decreasing part of the loading diagram indicates that there is an instability in the force-controlled loading. In Fig. 4 we present dependence of the total energy $\Psi$ on $F$, where

$$
\Psi \quad \int_{0}^{b} \rho \psi r d r
$$

Now we consider the quasistatic deformation. In this case the kinetic equation takes the form

$$
\frac{d a}{d t} \quad \hat{\mathcal{F}}(a ; F)
$$

Following Eremeyev and Pietraszkiewicz (2009) we assume the constant loading rate. This means that $F F_{0} t$ for loading and $F \quad F_{0} t$ for unloading. Here $F_{0}$ describes the constant loading velocity. Using substitution $t \quad F / F_{0}$ or $t \quad F / F_{0}$ we can transform (68) to the form

$$
\frac{d a}{d F} \quad \mp \hat{\mathcal{F}}(a ; F),
$$

where sign (-) is used for the loading process while $(+)$ for unloading, and the kinetic factor $k$ is replaced by $\hat{k} \quad k / F_{0}$.

Further we restrict ourselves to the solution when the new phase nucleates in the centre of the plate. In this case the initial data for (69) are $a\left(F_{1}^{*}\right) \quad 0$ for loading and $a\left(F_{2}^{*}\right) \quad 1$ for unloading. The loading diagrams are presented in Figs. 5-7. In Fig. 5 we assume $\xi \quad 0$ and $\varsigma_{0} \quad 0$. The shape and size of the hysteresis loop depend on the parameter $\hat{k}$ alone. The dashed segment $A B$ depicts the equilibrium part of the diagram. When $\hat{k}$ increases the area of hysteresis loop decreases. Examples of several deformation paths for different values of $\hat{k}$ are given in Fig. 5, see the loops $A B^{\prime} B A^{\prime}, A B^{\prime \prime} B A^{\prime \prime}, A B^{\prime \prime \prime} B A^{\prime \prime \prime}$, etc. With the growing $\hat{k}$ we obtain the narrowing loops. The limit $\hat{k} \rightarrow \infty$ corresponds both to the infinitely large kinetic factor $k$ and to the infinitely small loading velocity $F_{0}$. In the limit $\hat{k} \rightarrow \infty$ the hysteresis loop reduces to the equilibrium segment $A B$.

In Fig. 6 we use non-zero values of $\varsigma_{0}$ but again assume $\xi \quad 0$. In this case the equilibrium segments of the loading diagram can be found from the equations $\varsigma \quad \pm \varsigma_{0}$. The phase transformation begins when $\varsigma \quad \varsigma_{0}$ for loading and $\varsigma \quad \varsigma_{0}$ for unloading. Hence, in the thermodynamic equilibrium we have the parallelogram $A^{\prime} B^{\prime} B^{\prime \prime} A^{\prime \prime}$. The size of hysteresis loop increases with the increase of $\varsigma_{0}$ and, as in the previous case, depends on $\hat{k}$.

In Fig. 7 we present the hysteresis loops in the general case of the kinetic function, i.e. we use non-zero values of $\varsigma_{0}$ and $\xi$ in (51). Since $\left.\mathcal{F}(\varsigma)\right|_{\xi} \quad 0>\left.\mathcal{F}(\varsigma)\right|_{\xi \neq 0}$, the phase interface velocity $V \quad d a / d t$ is lower in the case of $\xi \quad 0$. Hence, the size of hysteresis loop is smaller in the case when $\xi \neq 0$. In Fig. 7 the hysteresis loops are given at the same values of $\hat{k}$ as in the previous case, but with $\xi \neq 0$. The grey region in Fig. 7 denotes the region of the maximal hysteresis loop shown in Fig. 6 . In the thermodynamic equilibrium we have again the parallelogram $A^{\prime} B^{\prime} B^{\prime \prime} A^{\prime \prime}$ as a limit of the hysteresis loops. Influence of the parameter $\xi$ is more pronounced far from the thermodynamic equilibrium and for dynamic processes. 


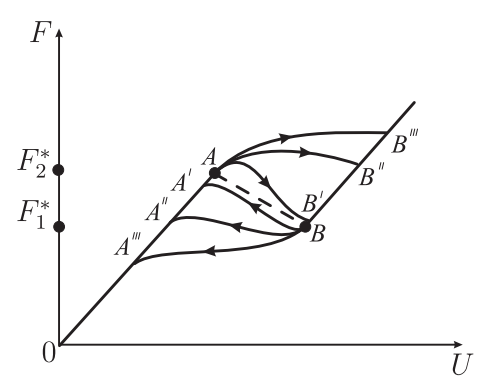

Fig. 5. $F U$ diagram for the two phase plate, $\xi=0, \varsigma_{0}=0$, and the kinetic function $\mathcal{F}=k \varsigma$.

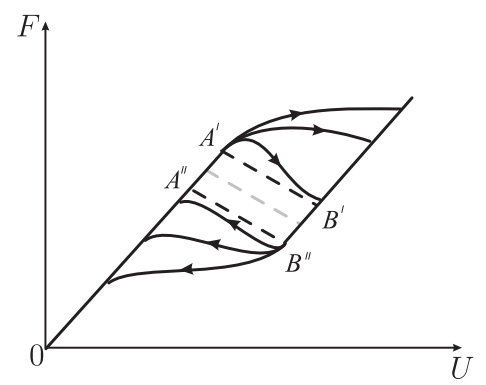

Fig. 6. $F U$ diagram for the two phase plate, $\xi=0$, and the kinetic function becomes as in Eremeyev and Pietraszkiewicz (2009).

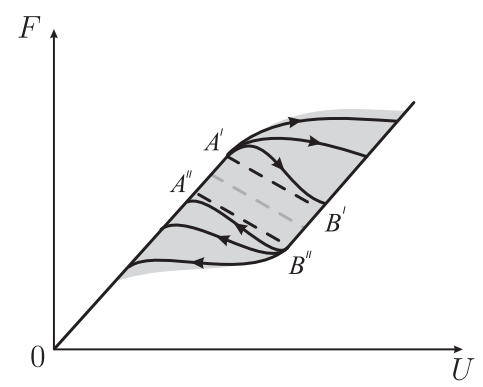

Fig. 7. $F \quad U$ diagram for the two phase plate, $\mathcal{F}$ takes the form (51). The grey region denotes the maximal hysteresis loop shown in Fig. 6 .

\subsection{Stretching and bending}

Let us consider stretching and bending of the elastic plate given in Fig. 1 with one coherent interface. We assume again that $\varphi \quad 0$. In general, if $\varphi \neq 0$ then bending of a thermoelastic plate appears, but for thin plates this influence is negligible for $q \neq 0$. In the linear theory of plates the boundary-value problems for the in-plane deformation and for the deflection can be solved independently. But for the plate with a phase interface the kinetic Eq. (50) is non-linear. Moreover, the characteristic feature of PT is the transformation strain which creates in-plane strains and stresses. Hence, for the plate undergoing PT bending and stretching problems are coupled, in general. The translation and rotation fields are given by

$$
\begin{array}{ll}
w & w_{0}+\frac{c_{1} r^{2}}{2}+c_{2} \ln r \frac{q r^{4}}{64 \beta_{3}} \frac{q r}{2 \alpha_{4}}, \\
u & d_{1} r+\frac{d_{2}}{r}, \quad \vartheta \quad c_{1} r+\frac{c_{2}}{r} \frac{q r^{3}}{16 \beta_{3}} .
\end{array}
$$

Substituting these formulas into (63) and (64) we obtain values of the integration constants $w_{0}, c_{1}, c_{2}, d_{1}$, and $d_{2}$ for both phases. It can be proved that $w(r)$ and $w^{\prime}(r)$ are continuous function, so $\varsigma$ becomes $\varsigma \quad \llbracket \rho \psi \quad N_{r r} u^{\prime} \rrbracket M_{\phi r} \kappa_{p}$.

As in the previous case, we begin from the thermodynamic equilibrium. Now we have two loading parameters, $F$ and $q$, and $q$ influences the phase transformation, in general. At the initial moment $F \quad 0$ and $q \quad 0$, and the plate consists of one phase $A$. When $F$ attains the value $F_{1}^{*}(q)$ the new phase $B$ nucleates in the centre of the plate. Here the value $F_{1}^{*}(q)$ decreases linearly with increase of $q$, so that there exists the value $q^{*}$ such that the new phase appears at $F \quad 0$, i.e. $F_{1}^{*}(0) \quad F_{1}^{*}$ and $F_{1}^{*}\left(q^{*}\right) \quad$ 0. Dependence of the phase interface radius $a$ on $F$ for different values of $q$ is given in Fig. 8(a), while dependence of $a$ on $q$ is shown in Fig. 8(b). When $F$ attains the value $F_{2}^{*}$ the new phase occupies the whole plate so that for $F>F_{2}^{*}$ the plate consists entirely of the phase $B$. In our model $F_{2}^{*}$ does not depend on $q$. On the $F \quad q$ diagram there is the region where the two-phase state of the plate exists, see Fig. 9. It is clear that non-zero values of transverse loads $q$ extend the region where the phase transformation is possible. On the other hand, the influence of $F$ is more significant than the influence of $q$. 

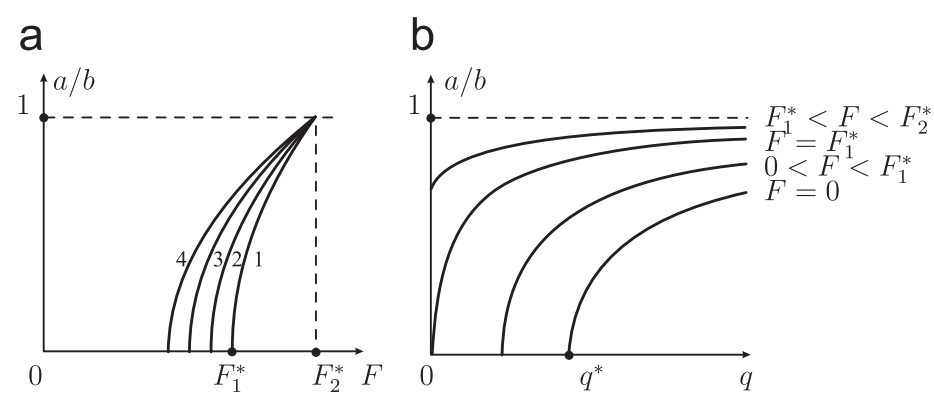

Fig. 8. Dependence of the phase interface radius $a$ on the external loads: (a) $a$ vs $F$ for different values of $q$, curves 14 relate to $0=q_{1}<q_{2}<q_{3}<q_{4}$ and (b) $a$ vs $q$ for different values of $F$.

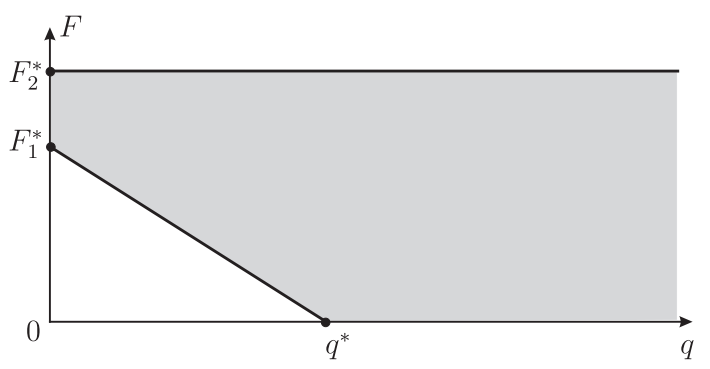

Fig. 9. Region on the $F \quad q$ diagram where the two phase state exists.

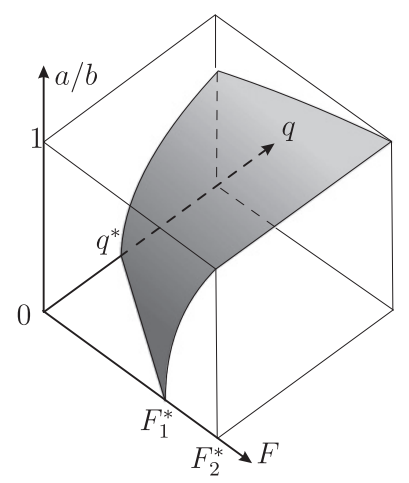

Fig. 10. Dependence of the phase interface radius $a$ on $F$ and $q$.

For example, $F_{1}^{*} \ll b q^{*}$ for reasonable values of the material parameters, so one needs to apply higher values of $q$ to reach the two-phase state. Hence, we can say that $F$ is the primary loading parameter which is responsible for the phase transformation. In Fig. 10 we present $a$ as the function of two variables, $F$ and $q$.

Since $q$ changes the value of $F$ when the new phase nucleates, the loading diagram changes as well. In Fig. 11 (a), $F \quad U$ diagrams are given for different values of $q$. The segments $A B, A B^{\prime}$, and $A B^{\prime \prime}$ correspond to different values of $q$ and different two-phase states of the plate. The ordinate of the point $A$ is equal to $F_{2}^{*}$, and this value does not depend on $q$, while the ordinates of the points $B, B^{\prime}$, and $B^{\prime \prime}$ are $F_{1}^{*} F_{1}^{*}(0), F_{1}^{(1)^{*}} F_{1}^{* *}\left(q_{1}\right), F_{1}^{(2)^{*}} \quad F_{1}^{*}\left(q_{2}\right)$ with $q_{1}<q_{2}$. Thus, the increase of $q$ leads to the increase of angle of the segment $A B, A B^{\prime}$, etc.

For quasistatic processes the kinetic equation takes the form

$$
\frac{d a}{d t} \hat{\mathcal{F}}(a ; F, q) \text {. }
$$

Since $F$ is recognised as the primary parameter responsible for the phase transformation, let us consider again the constant loading rate for $F$ with constant $q$. This means that we consider $q$ as a parameter. Assuming $F \quad F_{0} t$ for loading and $F \quad F_{0} t$ for unloading, we can transform (70) to the form

$$
\frac{d a}{d F} \quad \mp \hat{\mathcal{F}}(a ; F, q),
$$

where $k$ is replaced by $\hat{k} \quad k / F_{0}$. 

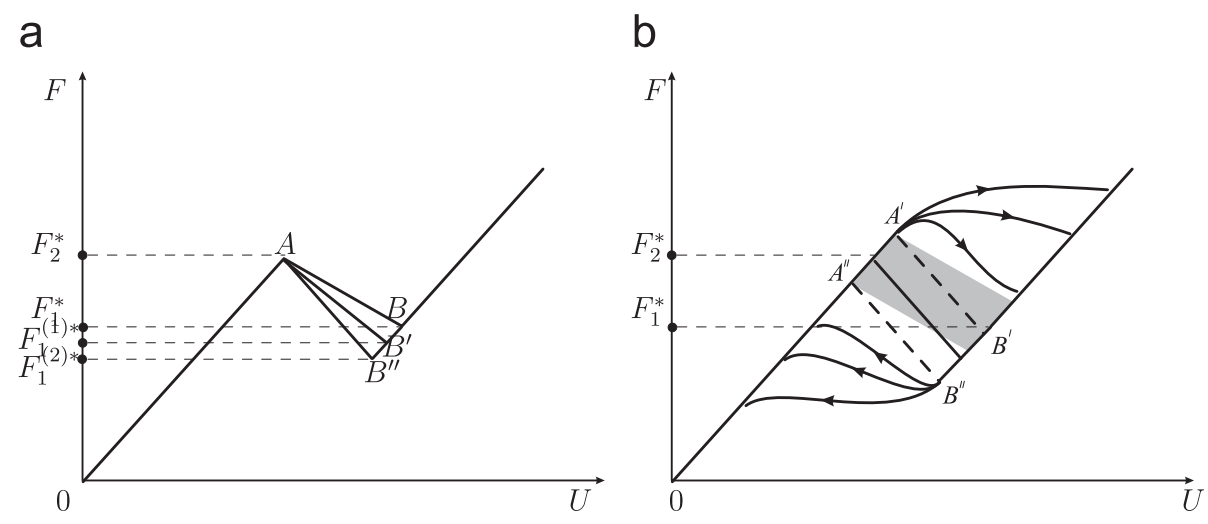

Fig. 11. (a) Equilibrium $F \quad U$ diagrams for the two phase plate, $F_{1}^{(1) *}=F_{1}^{*}\left(q_{1}\right), F_{1}^{(2) *}=F_{1}^{*}\left(q_{2}\right)$ with $q_{1}<q_{2}$, and (b) $F \quad U$ diagrams for the two phase plate under quasistatic loading with $q \neq 0$ and $\xi=0$.

The loading diagrams for $q \neq 0$ are presented in Fig. 11(b). Here we assume that $\xi \quad 0$ but $\varsigma \neq 0$. The dashed lines denote the equilibrium part $A^{\prime} B^{\prime} B^{\prime \prime} A^{\prime \prime}$ of the loading diagram which are limits when $\hat{k} \rightarrow \infty$. The grey rectangle shows the equilibrium part if $q \quad 0$. For $q \neq 0$ the size of the hysteresis loop is greater than in the case $q \quad 0$, in general.

The simple examples considered above demonstrate that the boundary-value problem for the two-phase plate can be solved within the framework of the general theory of thermoelastic shells presented in the previous sections and in Eremeyev and Pietraszkiewicz (2009). Let us note some features of the solutions. As in 3D case, the 2D problem discussed here has non-unique solutions, in general. Moreover, we have assumed that there exists only one phase interface, although even in the axisymmetric deformation one can consider two or more interfaces. In particular, the experimental data of He and Sun (2010a,b) show that the number of interfaces depends on the loading rate and thermal effects. We also note that the solution is sensitive to the problem parameters, such as the elasticity moduli, transformation strains, and the kinetic function.

\section{Discussion and conclusions}

As we have mentioned in Introduction, the literature on experimental investigations of thin-walled structures made of materials undergoing PT is extensive. The stress-induced PT are widely observed in superelastic shape memory alloys (SMA) and shape memory polymers, such as NiTi, NiMnGa, AgCd, AuCd, CuAlNi, polyurethane, etc. Here we briefly discuss some peculiarities of PT in thin-walled structures and their influence on the problem statements within 2D thermomechanics of two-phase shells.

The standard shape of specimen used in experiments is similar to the shape of plate, strip, band, or tube. Thin plates or strips made of SMA are widely used in experimental mechanics. Tension of the SMA plate is studied in many works applying various techniques, see e.g. Vivet and Lexcellent (2001), Lexcellent et al. (2002), Pieczyska et al. (2005, 2006a,b), Daly et al. (2007), Tobushi et al. (2009), Zhang et al. (2010), He and Sun (2010a,b) and Pieczyska (2010). In particular, optical and infrared techniques, the profilometry, etc. are used. Tension and torsion of a thin SMA strip was investigated by Tobushi et al. (2009). Tension and torsion of SMA microtubes are also widely investigated to understand the behaviour of martensitic materials, see Siddons and Moon (2001), Li and Sun (2002), Sun and Li (2002), Sittner et al. (2003), Feng and Sun (2006, 2007), Ng and Sun (2006), Favier et al. (2007), Schlosser et al. (2007), Wang et al. (2007), Buenconsejo et al. (2008), Lagoudas (2008), He and Sun (2009a,b) and Mao et al. (2010). These experiments demonstrate the macroscopic domain of new phase formation, its evolution during loading and annihilation after unloading. In the case of strips new phase forms as a few bands across the strip. In the case of tubes the new phase may appear as a helical band which width and shape depend on acting loads. The cylindrical bands are also observed, see Li and Sun (2002) and Ng and Sun (2006). The cylindrical bands and their evolution during loading were considered analytically by Eremeyev and Pietraszkiewicz (2009) applying the shell theory. The phase boundary between "old" and "new" phases, for example austenite-martensite phases, in many cases can be interpreted as a coherent sharp phase interface.

Another interesting example of PT in thin-walled structures are tents and tunnels discovered and investigated in detail in Bhattacharya et al. (1999), Hane (1999), James and Hane (2000), Shu (2000, 2002) and Bhattacharya and James (2005). For example, tent- or dome-shaped structures appear in martensitic thin films during PT. Each tent consists of four triangles of a new phase with different crystal lattices separated by four edges. These edges can be considered as the phase interfaces incoherent in rotations. It was shown that these two-phase structures can be energetically preferable in comparison with other one- and two-phase stressed states.

Summarising the peculiarities of PT in thin-walled structures described in works mentioned above, we have found that:

- Thin-walled elements made of SMA demonstrate the similar behaviour as the 3D elements with PT, i.e. there exist hysteresis loops, the reversible pseudoelasticity, etc. On the other hand, the behaviour of thin-walled structures may 
differ from the one of bulk bodies. In thin structures some possible transformations related to 3D PT are forbidden due to geometric restrictions, see Bhattacharya (2003) for details. Also differences in the microstructure influence PT; for example, depending on the method of production the columnar structures are observed in thin films but not in bulk bodies, see Miyazaki et al. (2009).

- PT is highly sensitive to the value of loading and rate of the loading. For example, the number of new phase strips in microtubes increases with the rate of tension, see Zhang et al. (2010).

- The temperature evolution plays a significant role in the new phase formation and the phase interface evolution. In particular, the nonhomogeneous temperature field due to PT are used by Pieczyska et al. (2006a, 2009); Pieczyska (2010) for the visualisation of PT. Dependence of number of bands in microtubes on the rate of loading can be explained as the transition from the isothermal loading to adiabatic one, see Zhang et al. (2010) and He and Sun (2010b).

- Depending on the loading type there are two possibilities in PT: the homogeneous deformations or nonhomogeneous ones with the formation of macroscopic phase interfaces and of bands consisting of the new phase. For example, Sun and $\mathrm{Li}$ (2002) has shown that torsion leads to the homogeneous deformation while tension results in the helical band formation. In the general case, the results of action of biaxial loadings (torsion and tension) on PT depends on the values of torsional and tensional forces.

- Although the microstructure near the phase interface can be very complicated, see e.g. Bhattacharya (2003) and Bhattacharya and James (2005), one can use the sharp interface model by introducing the effective surface energy density as in Stupkiewicz (2007) and Petryk and Stupkiewicz (2010). PT in shells with the line tension were considered by Pietraszkiewicz et al. (2007).

- PT are sensitive to various imperfections, boundary conditions and other parameters, in general.

- Such thin structures as tunnels, tents, and thin films demonstrate, in general, large deflections (in comparison with thickness) and rotations with possible wrinkling.

Thin plates, strips, and tubes made of SMA are used not only as specimens, but also as working elements of microelectromechanical systems (MEMS), see e.g. Shu (2002), Bhattacharya and James (2005) and Tobushi et al. (2009), and books by Bhattacharya (2003), Lagoudas (2008) and Miyazaki et al. (2009).

In order to model these peculiarities of behaviour of PT in thin-walled structures, one needs to develop the general thermomechanical theory taking into account the non-linear deformations during PT, large translations and rotations, and hence the geometrical nonlinearity, the temperature field evolution, the anisotropy of phases., etc. We believe that to meet all requirements the consistent 2D thermomechanical model of PT should be formulated in the most general form using the resultant 2D non-linear shell theory (Libai and Simmonds, 1983, 1998; Chróścielewski et al., 2004) with appropriate resultant 2D thermodynamics. Applying the thermodynamic driving force and using kinetics of the interface, the new phase domain morphology and its evolution can appropriately be modelled by the sharp interface model. Such 2D model has definite advantages with respect to 3D modelling, because this allows one to avoid solving the complicated 3D boundary-value problems of thermomechanics with PT in thin regions.

In this paper we have developed the resultant, two-dimensional thermomechanics of shells undergoing diffusionless, displacive phase transitions of martensitic type of the shell material, which meets all the requirements mentioned above. In the formulation we have extended our previous (Eremeyev and Pietraszkiewicz, 2009, 2010) resultant surface entropy inequality by completing it with the referential surface temperature deviation field and its dual - the referential surface entropy deviation field - as well as with some extra surface fields related to those deviation fields. We have also provided the corresponding extended constitutive equations for thermoelastic and thermoviscoelastic shells of differential type.

Along the curvilinear phase interface we have derived appropriate thermodynamic continuity condition and have proposed the corresponding kinetic equation, which allow one to determine position and quasistatic motion of the interface relative to the base surface.

The above resultant thermomechanic shell model with PT has been illustrated by two axisymmetric numerical examples of stretching and bending of the circular plate undergoing phase transition. The numerical results indicate that: (a) as in the 3D theory of phase transformations in elastic bodies, the solution is non-unique, in general; (b) presence of decreasing branch of the loading diagram indicates existence of instability during the force-controlled loading; (c) the quasistatic loading diagrams contain hysteresis loops which shape and size depend on the material parameters, kinetic function and loading rate; and (d) the in-plane and out-of-plane deformations of the plate undergoing PT are coupled due to the non-linear kinetic equation and the phase transformation strains.

In order to apply our resultant 2D thermodynamical model of PT for verification of realistic 2D experimental observations on thin-walled samples presented in papers cited above, one still needs to develop 2D numerical codes based on extended finite element method (XFEM) for shells with moving singular curves. This poses a serious challenge for the next years.

\section{Acknowledgement}

After this paper was submitted to JMPS, we received the draft of paper by Prof. J.G. Simmonds submitted to J. Elasticity (Simmonds, in press), where a resultant shell thermomechanics was presented. After email discussion we all concluded that, despite of differences in notation, sign convention, and definitions of some 2D compound fields, the resultant 2D 1st 
and 2nd Laws of both papers are essentially the same for the quasistatic processes in regular shells. We would like to thank Prof. J.G. Simmonds for this thorough and deep research discussion.

The first author was supported by the RFBR with the Grant no. 09-01-00459 and by the DFG with the Grant no. AL 341/33-1, while the second author by the Polish Ministry of Science and Higher Education with the Grant no. N501 007333.

\section{References}

Abeyaratne, R., Knowles, J.K., 2006. Evolution of Phase Transitions: A Continuum Theory. Cambridge University Press, Cambridge.

Agrawal, A., Steigmann, D.J., 2008. Coexistent fluid phase equilibria in biomembranes with bending elasticity. Journal of Elasticity 93 (1), 6380.

Berezovski, A., Engelbrecht, J., Maugin, G.A., 2008. Numerical Simulation of Waves and Fronts in Inhomogeneous Solids. World Scientific, New Jersey. Bhattacharya, K., 2003. Microstructure of Martensite: Why It Forms and How It Gives Rise to the Shape Memory Effect. Oxford University Press, Oxford.

Bhattacharya, K., DeSimone, A., Hane, K.F., James, R.D., Palmstrøm, C.J., 1999. Tents and tunnels on martensitic films. Materials Science and Engineering A 273 (Sp. Iss. SI), 685689.

Bhattacharya, K., James, R.D., 1999. A theory of thin films of martensitic materials with applications to microactuators. Journal of the Mechanics and Physics of Solids 47 (3), 531576.

Bhattacharya, K., James, R.D., 2005. The material is the machine. Science 307 (5706), 5354.

Boulbitch, A.A., 1999. Equations of heterophase equilibrium of a biomembrane. Archive of Applied Mechanics 69 (2), 8393.

Buenconsejo, P.J.S., Ito, K., Kim, H.Y., Miyazaki, S., 2008. High strength superelastic Ti Ni microtubes fabricated by sputter deposition. Acta Materialia 56 (9), 20632072.

Chróścielewski, J., Makowski, J., Pietraszkiewicz, W., 2004. Statics and Dynamics of Multi Shells: Nonlinear Theory and Finite Element Method (in Polish). Wydawnictwo IPPT PAN, Warszawa.

Coleman, B.D., Noll, W., 1963. The thermodynamics of elastic materials with heat conduction and viscosity. Archive for Rational Mechanics and Analysis $13(1), 167178$.

Courant, R., Hilbert, D., 1989. Methods of Mathematical Physics. Vol. II. Partial Differential Equations. Wiley, New York.

Daly, S., Ravichandran, G., Bhattacharya, K., 2007. Stress induced martensitic phase transformation in thin sheets of Nitinol. Acta Materialia 55 (10), 35933600.

Elliott, C.M., Stinner, B., 2010. A surface phase field model for two phase biological membranes. SIAM Journal of Applied Mathematics 70 (8), 29042928.

Eremeyev, V.A., Pietraszkiewicz, W., 2004. The non linear theory of elastic shells with phase transitions. Journal of Elasticity 74 (1), 6786.

Eremeyev, V.A., Pietraszkiewicz, W., 2006. Local symmetry group in the general theory of elastic shells. Journal of Elasticity 85 (2), 125152.

Eremeyev, V.A., Pietraszkiewicz, W., 2009. Phase transitions in thermoelastic and thermoviscoelastic shells. Archives of Mechanics 61 (1), 4167.

Eremeyev, V.A., Pietraszkiewicz, W., 2010. On tension of a two phase elastic tube. In: Pietraszkiewicz, W., Kreja, I. (Eds.), Shell Structures: Theory and Applications, vol. 2. CRC Press, Boca Raton, pp. 6366.

Eremeyev, V.A., Zubov, L.M., 1991. On the stability of equilibrium of nonlinear elastic bodies with phase transformations (in Russian). Izv. USSR Academy of Sciences. Mekhanika Tverdogo Tela (Mechanics of Solids) (2), 5665.

Eremeyev, V.A., Zubov, L.M., 2008. Mechanics of Elastic Shells (in Russian). Nauka, Moscow.

Favier, D., Louche, H., Schlosser, P., Orgeas, L., Vacher, P., Debove, L., 2007. Homogeneous and heterogeneous deformation mechanisms in an austenitic polycrystalline $\mathrm{Ti} 50.8$ at.\% $\mathrm{Ni}$ thin tube under tension. Investigation via temperature and strain fields measurements. Acta Materialia 55 (16), 53105322.

Feng, P., Sun, Q.P., 2006. Experimental investigation on macroscopic domain formation and evolution in polycrystalline NiTi microtubing under mechanical force. Journal of the Mechanics and Physics of Solids 54 (8), 15681603.

Feng, P., Sun, Q.P., 2007. In situ profilometry for non uniform strain field measurement of NiTi shape memory alloy microtubing under complex stress states. Smart Materials \& Structures 16 (1), S179 S186.

Fischer, F.D. (Ed.), 2004. Moving Interfaces in Crystalline Solids. CISM Cources and Lectures No 543. Springer, Wien.

Freidin, A.B., Fu, Y.B., Sharipova, L.L., Vilchevskaya, E.N., 2006. Spherically symmetric two phase deformations and phase transition zones. International Journal of Solids and Structures 43 (14 15), 44844508.

Gibbs, J.W., 1928. On the equilibrium of heterogeneous substances. In: The Collected Works of J. Willard Gibbs. Longmans. Green \& Co, New York, pp. 55353.

Green, A.E., Naghdi, P.M., 1970. Non isothermal theory of rods, plates and shells. International Journals of Solids and Structures 6 (2), 635648.

Green, A.E., Naghdi, P.M., 1979. On thermal effects in the theory of shells. Proceedings of the Royal Society of London Series A 365 (1721), 161190.

Grinfeld, M., 1991. Thermodynamics Methods in the Theory of Heterogeneous Systems. Longman, Harlow.

Gurtin, M.E., 1993. Thermomechanics of Evolving Phase Boundaries in the Plane. Clarendon Press, Oxford.

Gurtin, M.E., 2000. Configurational Forces as Basic Concepts of Continuum Physics. Springer Verlag, Berlin.

Hane, K.F., 1999. Bulk and thin film microstructures in untwinned martensites. Journal of the Mechanics and Physics of Solids 47, 19171939.

He, Y.J., Sun, Q.P., 2009a. Effects of structural and material length scales on stress induced martensite macro domain patterns in tube configurations. International Journal of Solids and Structures 46 (16), 30453060.

He, Y.J., Sun, Q.P., 2009b. Scaling relationship on macroscopic helical domains in NiTi tubes. International Journal of Solids and Structures 46 (24), 42424251.

He, Y.J., Sun, Q.P., 2010a. Macroscopic equilibrium domain structure and geometric compatibility in elastic phase transition of thin plates. International Journal of Mechanical Sciences 52 (2), 198211.

He, Y.J., Sun, Q.P., 2010b. Rate dependent domain spacing in a stretched NiTi strip. International Journal of Solids and Structures 47 (20), 27752783.

James, R.D., Hane, K.F., 2000. Martensitic transformations and shape memory materials. Acta Materialia 48 (1), 197222.

James, R.D., Rizzoni, R., 2000. Pressurized shape memory thin films. Journal of Elasticity 59 (1 3), 399436.

Kienzler, R., Herrman, G., 2000. Mechanics in Material Space with Applications to Defect and Fracture Mechanics. Springer Verlag, Berlin.

Konopińska, V., Pietraszkiewicz, W., 2007. Exact resultant equilibrium conditions in the non linear theory of branched and self intersecting shells. International Journals of Solids and Structures 44 (1), 352369.

Lagoudas D.C. (Ed.), 2008. Shape Memory Alloys: Modeling and Engineering Applications. Springer, Berlin.

Lexcellent, C., Vivet, A., Bouvet, C., Calloch, S., Blanc, P., 2002. Experimental and numerical determinations of the initial surface of phase transformation under biaxial loading in some polycrystalline shape memory alloys. Journal of the Mechanics and Physics of Solids 50 (12), 27172735.

Li, Z.Q., Sun, Q.P., 2002. The initiation and growth of macroscopic martensite band in nano grained NiTi microtube under tension. International Journal of Plasticity 18 (11), 14811498.

Libai, A., Simmonds, J.G., 1983. Nonlinear elastic shell theory. Advances of Applied Mechanics 23, 271371.

Libai, A., Simmonds, J.G., 1998. The Nonlinear Theory of Elastic Shells, second ed. Cambridge University Press, Cambridge.

Makowski, J., Pietraszkiewicz, W., 2002. Thermomechanics of shells with singular curves. Zesz. Nauk. No 528/1487/2002, IMP PAN, Gdańsk.

Mao, S.C., Luo, J.F., Zhang, Z., Wu, M.H., Liu, Y., Han, X.D., 2010. EBSD studies of the stress induced B2 B19“ martensitic transformation in NiTi tubes under uniaxial tension and compression. Acta Materialia 58 (9), 33573366.

Maugin, G.A., 1993. Material Inhomogeneities in Elasticity. Chapman Hall, London. 
Maugin, G.A., 1998. On shock waves and phase-transition fronts in continua. ARI—An International Journal for Physical and Engineering Sciences 50 (3), $141-150$.

Miyazaki, D.C., Fu, Y.Q., Huang, W.M. (Eds.), 2009. Thin Film Shape Memory Alloys: Fundamentals and Device Applications. Cambridge University Press, Cambridge.

Murdoch, A.I., 1976a. On the entropy inequality for material interfaces. ZAMP 27 (5), 599-605.

Murdoch, A.I., 1976b. A thermodynamical theory of elastic material interfaces. The Quarterly Journal of Mechanics and Applied Mathematics 29 (3), $245-274$.

Muschik, W., 2008. Survey of some branches of thermodynamics. Journal of Non-Equilibrium Thermodynamics 33 (2), 165-198

Muschik, W., Papenfuss, C., Ehrentraut, H., 2001. A sketch of continuum thermodynamics. Journal of Non-Newtonian Fluid Mechanics 96 (1-2, Sp. Iss. SI), 255-290.

Ng, K.L., Sun, Q.P., 2006. Stress-induced phase transformation and detwinning in NiTi polycrystalline shape memory alloy tubes. Mechanics of Materials 38 (1-2, Sp. Iss. SI), 41-56.

Petryk, H., Stupkiewicz, S., 2010. Interfacial energy and dissipation in martensitic phase transformations. Part I: theory. Journal of the Mechanics and Physics of Solids 58 (3), 390-408.

Pieczyska, E., 2010. Activity of stress-induced martensite transformation in TiNi shape memory alloy studied by infrared technique. Journal of Modern Optics 57 (18, Sp. Iss. SI), 1700-1707.

Pieczyska, E., Gadaj, S., Nowacki, W.K., Hoshio, K., Makino, Y., Tobushi, H., 2005. Characteristics of energy storage and dissipation in TiNi shape memory alloy. Science and Technology of Advanced Materials 6 (8), 889-894.

Pieczyska, E.A., Gadaj, S.P., Nowacki, W.K., Tobushi, H., 2006a. Phase-transformation fronts evolution for stress- and strain-controlled tension tests in TiNi shape memory alloy. Experimental Mechanics 46 (4), 531-542.

Pieczyska, E.A., Nowacki, W.K., Tobushi, H., Hayashi, S., 2009. Thermomechanical properties of shape memory polymer subjected to tension in various conditions. QIRT Journal 6 (2), 189-205.

Pieczyska, E.A., Tobushi, H., Gadaj, S.P., Nowacki, W.K., 2006b. Superelastic deformation behaviors based on phase transformation bands in TiNi shape memory alloy. Materials Transactions 47 (3), 670-676.

Pietraszkiewicz, W., Chróścielewski, J., Makowski, J., 2005. On dynamically and kinematically exact theory of shells. In: Pietraszkiewicz, W., Szymczak, C. (Eds.), Shell Structures: Theory and Applications. Taylor \& Francis, London, pp. 163-167.

Pietraszkiewicz, W., Eremeyev, V.A., Konopińska, V., 2007. Extended non-linear relations of elastic shells undergoing phase transitions. ZAMM 87 (2), 150-159.

Romano, A., 1993. Thermodynamics of Phase Transitions in Classical Field Theory. World Scientific, Singapore.

Rubin, M.B., 2004. Restrictions on linear constitutive equations for a rigid heat conducting Cosserat shell. International Journal of Solids and Structures 41 (24-25), 7009-7033.

Rubin, M.B., 2006. Heat conduction between confocal elliptical surfaces using the theory of a Cosserat shell. International Journal of Solids and Structures 43 (2), 295-306.

Schlosser, P., Louche, H., Favier, D., Orgeas, L., 2007. Image processing to estimate the heat sources related to phase transformations during tensile tests of NiTi tubes. Strain 43 (3), 260-271.

Shu, Y.C., 2000. Heterogeneous thin films of martensitic materials. Archive of Rational Mechanics and Analysis 153 (1), 39-90.

Shu, Y.C., 2002. Shape-memory micropumps. Materials Transactions 43 (5, Sp. Iss. SI), 1037-1044.

Siddons, D.J., Moon, J.R., 2001. Tensile and compression performance of superelastic NiTi tubing. Materials Science and Technology 17 (9), $1073-1078$.

Simmonds, J.G., 1984. The thermodynamical theory of shells: descent from 3-dimensions without thickness expansions. In: Axelrad, E.K., Emmerling, F.A. (Eds.), Flexible Shells: Theory and Applications. Springer, Berlin, pp. 1-11.

Simmonds, J.G., 2005. A simple nonlinear thermodynamic theory of arbitrary elastic beams. Journal of Elasticity 81 (1), 51-62.

Simmonds, J.G. A classical, nonlinear thermodynamic theory of elastic shells based on a single constitutive assumption. Journal of Elasticity, in press. doi: 10.1007/s10659-010-9293-2.

Sittner, P., Hashimoto, K., Kato, M., Tokuda, M., 2003. Stress induced martensitic transformations in tension/torsion of CuAlNi single crystal tube. Scripta Materialia 48 (8), 1153-1159.

Steinmann, P., Häsner, O., 2005. On material interfaces in thermomechanical solids. Archive of Applied Mechanics 75 (1), 31-41.

Stupkiewicz, S., 2007. Micromechanics of contact and interface layers. Lecture Notes in Applied and Computational Mechanics, vol. 30. Springer, Berlin.

Sun, Q.P., 2002. Mechanics of Martensitic Phase Transformation in Solids. Kluwer, Dordrecht.

Sun, Q.P., Li, Z.Q., 2002. Phase transformation in superelastic NiTi polycrystalline micro-tubes under tension and torsion-from localization to homogeneous deformation. International Journal of Solids and Structures 39 (13-14), 3797-3809.

Timoshenko, S.P., Woinowsky-Krieger, S., 1985. Theory of Plates and Shells. McGraw Hill, New York.

Tobushi, H., Pieczyska, E.A., Nowacki, W.K., Sakuragi, T., Sugimoto, Y., 2009. Torsional deformation and rotary driving characteristics of SMA thin strip. Archives of Mechanics 61 (3-4), 241-257.

Truesdell, C., 1984. Rational Thermodynamics, second ed. Springer, New York.

Truesdell, C., Toupin, R., 1960. The classical field theories. In: Flügge, S. (Ed.), Handbuch der Physik, vol. III/1. Springer, Berlin, pp. 226-793.

Truesdell, C.A., 1966. The Elements of Continuum Mechanics. Springer, Berlin.

Truesdell, C.A., 1991. A First Course in Rational Continuum Mechanics. Academic Press, Boston.

Vivet, A., Lexcellent, C., 2001. CuZnAl single crystals pseudoelastic behaviour under biaxial tensile loading: observations and analysis. Journal de Physique IV 11 (PR4), 205-212.

Wang, Y.F., Yue, Z.F., Wang, J., 2007. Experimental and numerical study of the superelastic behaviour on NiTi thin-walled tube under biaxial loading. Computational Materials Science 40 (2), 246-254.

Yeremeyev, V.A., Freidin, A.B., Sharipova, L.L., 2007. The stability of the equilibrium of two-phase elastic solids. Journal of Applied Mathematics and Mechanics 71 (1), 61-84.

Zhang, X.H., Feng, P., He, Y.J., Yu, T.X., Sun, Q.P., 2010. Experimental study on rate dependence of macroscopic domain and stress hysteresis in NiTi shape memory alloy strips. International Journal of Mechanical Sciences 52 (12), 1660-1670.

Zhilin, P.A., 1976. Mechanics of deformable directed surfaces. International Journals of Solids and Structures 12 (9-10), 635-648. 